\title{
Measuring continuous-variable quantum entanglement with parametric-amplifier-assisted homodyne detection
}

\author{
Jiamin Li $\odot,{ }^{1}$ Yuhong Liu, ${ }^{1}$ Nan Huo, ${ }^{1}$ Liang Cui, ${ }^{1}$ Sheng Feng, ${ }^{2}$ Xiaoying Li $1,{ }^{1}{ }^{*}$ and Z. Y. Ou ${ }^{1,3, \dagger}$ \\ ${ }^{1}$ College of Precision Instrument and Opto-electronics Engineering, Key Laboratory of Opto-electronics Information Technology, \\ Ministry of Education, Tianjin University, Tianjin 300072, People's Republic of China \\ ${ }^{2}$ Beijing Institute of Radio Measurement, Beijing 100854, People's Republic of China \\ ${ }^{3}$ Department of Physics, Indiana University-Purdue University Indianapolis, Indianapolis, Indiana 46202, USA
}

(Received 6 September 2018; revised manuscript received 14 January 2020; accepted 6 April 2020; published 1 May 2020)

\begin{abstract}
The traditional method for measuring Einstein-Podolsky-Rosen-type continuous-variable quantum entanglement relies on balanced homodyne detections, which are sensitive to vacuum quantum noise coupled in through losses due to various factors such as detector quantum efficiency and mode mismatching between the detected field and the local oscillator. In this paper, we propose and analyze a measurement method, which is realized by assisting the balanced homodyne detections with a high-gain phase-sensitive parametric amplifier. The employment of the phase-sensitive amplifier helps us to tackle the vacuum quantum noise originating from detection losses. Moreover, because the high-gain phase-sensitive amplifier can couple two fields of different types, the proposed scheme can be used to reveal quantum entanglement between two fields of different types by using only one balanced homodyne detection. Furthermore, detailed analysis shows that in the multimode case, the proposed scheme is also advantageous over the traditional method. Such a measurement method should find wide applications in quantum information and quantum metrology involving measurement of continuous variables.
\end{abstract}

DOI: 10.1103/PhysRevA.101.053801

\section{INTRODUCTION}

Homodyne detection, which measures the quadraturephase amplitudes of light, plays a central role in continuousvariable $(\mathrm{CV})$ quantum information processing (QIP), from characterizing the $\mathrm{CV}$ entanglement to fulfilling various kinds of QIP tasks [1-12]. In particular, the measurement of CV entanglement between two parties usually relies on the joint measurement of two sets of balanced homodyne detectors (BHD), in which a 50:50 beam splitter is used to superpose the input field and a strong local oscillator (LO). However, the measurement performed by BHD is prone to losses such as propagation loss, less-than-unit quantum efficiency of detectors, and imperfect mode matching between the input field and LO. As a result, the observed quantum effect is smaller than what is anticipated, and the quantum advantage of entanglement is often hampered by these losses. So far, there have been some attempts for improving upon the measurement scheme of homodyne detection to achieve the loss tolerance through homodyne intensity correlation measurement of the quantum fields [13-15], but they were not widely used in quadrature amplitude measurement. As for the original BHD, it requires detectors with high efficiency which may not exist for some fields such as $2-\mu \mathrm{m}$ optical waves and $\mathrm{THz}$ waves. Entanglement between different waves such as optical and atomic

\footnotetext{
*xiaoyingli@tju.edu.cn

†zheyuou@tju.edu.cn
}

waves may exist in atomic Raman processes [16-18]. Such kind of entanglement cannot be revealed by BHD because of the lack of detection scheme for atoms. So it is hard to carry out investigations and applications of entangled fields in the wavelengths outside the mature detection technologies.

Recent investigation [19] shows that a high-gain phasesensitive amplifier (PSA) can be viewed as homodyne detection, in which the strong pump of PSA serves as the $\mathrm{LO}$ and squeezing generated from four-wave mixing (FWM) in fiber was successfully measured by scanning the pump phase of PSA. The results illustrated that the noise reduction measured with a direct intensity detection scheme by PSA has the advantage of detection loss tolerance. Indeed, the idea of using high-gain PSA to measure quantum noise reduction was first adopted by Flurin et al. to overcome the huge classical electronic noise in measuring the entanglement of microwaves [20], in which the PSA formed by Josephson mixer functions as a disentangler. The results in Refs. $[19,20]$ show that the inseparability of entanglement between two fields can be characterized by directly measuring the intensity at one output of PSA. This kind of method has two advantages. One is that it is tolerant to detection loss and the other is that only the measurement of one field is required. However, compared to the traditional method of measuring entanglement with two BHDs, the method of using PSA followed with a power detector for measuring noise reduction has the following deficiencies. First, the measured noise fluctuations of the quantum states in Refs. $[19,20]$ are determined by the power of PSA output. Since the input to the PSA is quantum fields of low 
photon numbers, the gain of PSA must be very high in order to have enough output power. The high pump power required for achieving high gain hinders the practicality. Second, the intensity directly measured at one output of PSA is the sum of noise fluctuations for both the quadrature-phase amplitudes $\hat{X}_{j}$ and $\hat{Y}_{j}(j=1,2)$ of two entangled fields, but the noise fluctuation of the quadrature amplitudes of two entangled fields, $\hat{X}_{1} \mp \hat{X}_{2}$ and $\hat{Y}_{1} \pm \hat{Y}_{2}$, cannot be individually measured. Therefore, this method, while it can be used to characterize the inseparability of entanglement, is not suitable for fulfilling many QIP tasks, such as quantum dense coding [1-4], teleportation [5-9], and quantum enhanced precision measurement [10-12].

In this paper, we propose and analyze a method realized by assisting the BHDs with a PSA. This method inherits the advantages of both BHDs and high-gain PSA in measuring $\mathrm{CV}$ entanglement. In addition to the aforementioned tolerance to detection loss and one-field detection for measuring entanglement between two fields, the quantum correlations between two entangled fields, such as $\left\langle\Delta^{2}\left(\hat{X}_{1} \mp \hat{X}_{2}\right)\right\rangle$ and $\left\langle\Delta^{2}\left(\hat{Y}_{1} \pm\right.\right.$ $\left.\left.\hat{Y}_{2}\right)\right\rangle$, are respectively and simultaneously measurable. Moreover, by studying the dependence of measurement upon the key parameters of this scheme, we find these advantages can be achieved by using a PSA with only moderate gain, which makes this method more practical.

The rest of the paper is organized as follows. In Sec. II, we briefly review the traditional method of measuring entanglement by using the joint measurement of two BHDs. In Sec. III, we introduce another type of homodyne detection realized by a high-gain PSA followed with a detector for directly measuring the intensity at one output of PSA, as done in Ref. [19]. In Sec. IV, we propose and investigate our measurement method realized by using PSA to assist the homodyne detection of one BHD as well as joint measurement by two BHDs. The simulation results obtained by varying the gain of PSA and the loss of BHD clearly demonstrate that the performance of this method surpasses those schemes in Secs. II and III, and the traditional method in Sec. II is just a specific case of this method for PSA with gain set to 1 . In Sec. V, we extend the measurement of entangled states from a single-temporal mode model to a multitemporal mode model, when the entangled state is generated from a pulse-pumped traveling-wave parametric amplifier with broadband spectrum [21-24]. The detailed analysis shows that this method is also advantageous over the traditional method in multimode cases. Finally, we conclude in Sec. VI.

\section{TRADITIONAL METHOD FOR MEASURING ENTANGLEMENT}

The Einstein-Podolsky-Rosen (EPR)-type entangled state was first put forward by Einsteinet al. in Ref. [25]. Defining $\hat{X}_{j}(\phi)=\hat{a}_{j} e^{-i \phi}+\hat{a}_{j}^{\dagger} e^{i \phi}(j=1,2)$ as the quadrature-phase amplitude of field $j$, where $\hat{a}_{j}^{\dagger}$ and $\hat{a}_{j}$ are the creation and annihilation operators of the field. For the conjugate observables $\hat{X}_{j} \equiv \hat{X}_{j}(0)$ and $\hat{Y}_{j} \equiv \hat{X}_{j}(\pi / 2),(j=1,2)$, although the commutation relation $\left[\hat{X}_{j}, \hat{Y}_{l}\right]=\delta_{j, l}(j, l=1,2)$ holds, quantum mechanics also has the commutation relation $\left[\hat{X}_{1}-\hat{X}_{2}, \hat{Y}_{1}+\right.$ $\left.\hat{Y}_{2}\right]=0$, which means that the quantum fluctuations for the difference and sum of quadrature amplitudes, $\hat{X}_{-}=\hat{X}_{1}-\hat{X}_{2}$

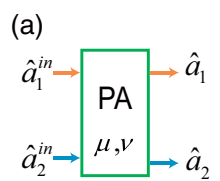

(b) Input

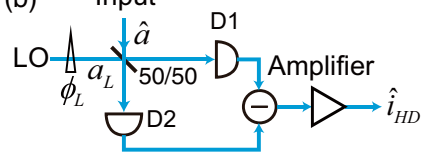

(c)

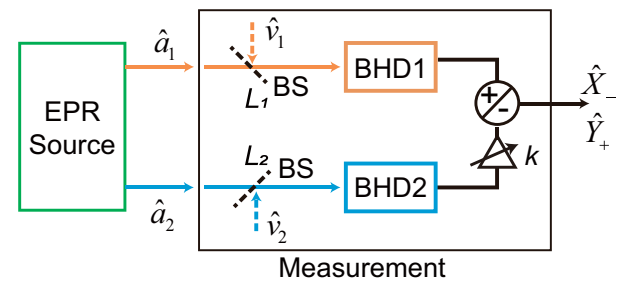

FIG. 1. (a) Entangled state generated from a parametric amplifier (PA). (b) The scheme of a balanced homodyne detection (BHD) system. (c) Traditional method of entanglement verification for Einstein-Podolsky-Rosen (EPR)-type entangled source. D1 and D2, detectors; LO, local oscillator. The detection loss of field $\hat{a}_{1} / \hat{a}_{2}$ is modeled as beam splitter (BS) of reflectivity $L_{1} / L_{2} . \hat{v}_{1} / \hat{v}_{2}$ represents the vacuum field coupled into the detected fields.

and $\hat{Y}_{+}=\hat{Y}_{1}+\hat{Y}_{2}$, can simultaneously approach zero. For the sake of convenience, in this paper, we will take the most common source of EPR state realized by a phase-insensitive parametric amplifier (PA) as an example [26,27].

\section{A. EPR states generated from parametric amplifiers}

Figure 1(a) shows the schematic of the entanglement source. The two input and output fields of a PA are labeled as $\hat{a}_{1,2}^{\text {in }}$ and $\hat{a}_{1,2}$, respectively, and the input fields are both in vacuum. Ideally, the input-output relations of the PA are given by

$$
\hat{a}_{1}=\mu \hat{a}_{1}^{\text {in }}+v \hat{a}_{2}^{\text {in } \dagger}, \hat{a}_{2}=\mu \hat{a}_{2}^{\text {in }}+v \hat{a}_{1}^{\text {in } \dagger},
$$

where $\mu, v$ with $\mu^{2}-v^{2}=1$ are the amplitude gains of the PA. The noise variances, $\hat{X}_{-}$and $\hat{Y}_{+}$, are expressed as $\left\langle\Delta^{2} \hat{X}_{-}\right\rangle,\left\langle\Delta^{2} \hat{Y}_{+}\right\rangle$and are both calculated to be $2(\mu-v)^{2}$. The corresponding shot noise limits (SNL), obtained by replacing the two fields $\hat{a}_{1}, \hat{a}_{2}$ with vacuum, are $\left\langle\Delta^{2} \hat{X}_{-}\right\rangle_{\mathrm{SNL}}=$ $\left\langle\Delta^{2} \hat{Y}_{+}\right\rangle_{\mathrm{SNL}}=2$. Therefore, the normalized noise reduction of the entangled source are

$$
\begin{aligned}
\left\langle\Delta^{2} \hat{X}_{-}\right\rangle_{s} & =\frac{\left\langle\Delta^{2} \hat{X}_{-}\right\rangle}{\left\langle\Delta^{2} \hat{X}_{-}\right\rangle_{\mathrm{SNL}}}=(\mu-v)^{2}<1, \\
\left\langle\Delta^{2} \hat{Y}_{+}\right\rangle_{s} & =\frac{\left\langle\Delta^{2} \hat{Y}_{+}\right\rangle}{\left\langle\Delta^{2} \hat{Y}_{+}\right\rangle_{\mathrm{SNL}}}=(\mu-v)^{2}<1,
\end{aligned}
$$

where subscript $s$ denotes normalization to SNL for the entangled source. The quantities $\left\langle\Delta^{2} \hat{X}_{-}\right\rangle_{s},\left\langle\Delta^{2} \hat{Y}_{+}\right\rangle_{s}$ also represent the noise reduction relative to SNL that is measured experimentally and is in the unit of $\mathrm{dB}$ in $\log$ scale. The entanglement verification for the two fields $\hat{a}_{1}$ and $\hat{a}_{2}$ is done through the inseparability criterion [28]:

$$
I_{s} \equiv\left\langle\Delta^{2} \hat{X}_{-}\right\rangle_{s}+\left\langle\Delta^{2} \hat{Y}_{+}\right\rangle_{s}<2=I_{s}^{\mathrm{SNL}},
$$

where $I_{s}^{\mathrm{SNL}}=2$ denotes the SNL of the inseparability quantity $I_{s}$, or the limit for the unentangled classical fields. So, the inseparability quantity $I_{s}$ can be considered as an entanglement measure. As $I_{s}$ is smaller, the degree of entanglement between 
the two fields is higher. From Eq. (2), we have $I_{s}=2(\mu-$ $v)^{2}<2$ for $v \neq 0$, satisfying the inseparability criterion in Eq. (3). For the case of $v \rightarrow \infty$, we have $I_{s} \rightarrow 0$, which means the two fields $\hat{a}_{1}$ and $\hat{a}_{2}$ are near perfectly correlated in the same way as the perfect EPR correlation [25] if the gain of PA is very high.

\section{B. Traditional method for entanglement measurement}

Traditionally, the EPR entangled state is characterized by the joint measurement of two balanced homodyne detectors [BHD, Fig. 1(b)]. As shown in Fig. 1(c), the quadraturephase amplitudes of the two entangled fields are respectively measured by BHD1 and BHD2. Each BHD consists of a 50:50 beam splitter (BS), two detectors (D1 and D2), and a subtracter followed with an electronic amplifier. The BS is used to superpose a strong local oscillator (LO) with a weak signal input field $\hat{a}$. The noise variance of the difference between the photocurrents of D1 and D2, usually analyzed by a spectrum analyzer, is expressed as

$$
\left\langle\Delta^{2} \hat{i}_{\mathrm{BHD}}\right\rangle=q^{2}\left|\alpha_{L}\right|^{2}\left\langle\Delta^{2} \hat{X}(\phi)\right\rangle,
$$

where $q$ is the gain of electronic amplifier and $\left|\alpha_{L}\right|$ is the amplitude of the LO. When the phase $\phi$ takes 0 and $\pi / 2$ by changing the phase of $\mathrm{LO}$, the photocurrent operator $\hat{i}_{\mathrm{BHD}}$ corresponds to measurement of quadrature amplitudes $\hat{X}$ and $\hat{Y}$, respectively.

The joint measurement of two fields [see Fig. 1(c)] is performed by using an electronic combiner. The subtraction and addition operation of the photocurrents of two BHDs give

$$
\begin{aligned}
& \hat{i}_{-}=\hat{i}_{1}-k \hat{i}_{2} \propto \hat{X}_{1}-k \hat{X}_{2}, \\
& \hat{i}_{+}=\hat{i}_{1}+k \hat{i}_{2} \propto \hat{Y}_{1}+k \hat{Y}_{2},
\end{aligned}
$$

when the phase $\phi$ of each BHD is locked at 0 and $\pi / 2$, respectively, where $k$ is the adjustable gain for optimizing the measurement. Similar to Eq. (2), the normalized variances of $\hat{i}_{-}$and $\hat{i}_{+}$to the vacuum input for $k=1$ are

$$
\begin{aligned}
& \left\langle\Delta^{2} \hat{i}_{-}\right\rangle_{N o r}=\left\langle\Delta^{2} \hat{X}_{-}\right\rangle_{s}=(\mu-v)^{2}, \\
& \left\langle\Delta^{2} \hat{i}_{+}\right\rangle_{N o r}=\left\langle\Delta^{2} \hat{Y}_{+}\right\rangle_{s}=(\mu-v)^{2} .
\end{aligned}
$$

Hence, the joint measurement of two BHDs gives the noise reduction quantities $\left\langle\Delta^{2} \hat{X}_{-}\right\rangle_{s}$ and $\left\langle\Delta^{2} \hat{Y}_{+}\right\rangle_{s}$ of the EPR source expressed in Eq. (2) as well as the inseparability quantity $I_{s}$.

\section{Challenges of the traditional measurement method}

From Eqs. (4) and (6), one sees that the role of strong LO in BHD is to increase the photocurrents of photodetectors and to effectively amplify a weak input signal field to a level that is otherwise buried in the classical noise such as detector's dark current, thermal electronic noise of current amplifiers, and ambient background light. However, the BHD cannot do much against vacuum noise coupled in through losses that inevitably exist in a real system, including less-than-unit quantum efficiency, imperfect propagation, and mode mismatching.

We can model the detection loss of BHD1 and BHD2 as BSs with reflectivity $L_{1}$ and $L_{2}$ [see Fig. 1(a)], respectively. The operators of the fields propagating though the BSs are given by $\hat{a}_{1,2}^{\prime}=\sqrt{1-L_{1,2}} \hat{a}_{1,2}+\sqrt{L_{1,2}} \hat{v}_{1,2}$, where $\hat{v}_{1,2}$ are the vacuum fields coupled into the detected fields through the BS's unused ports. For the sake of simplicity, we assume the detection losses of two BHDs are equal, i.e., $L_{1}=L_{2}=$ $L_{D}$. When the operators $\hat{a}_{1,2}$ in Eq. (1) are replaced with $\hat{a}_{1,2}^{\prime}$, after some algebra, the noise variance for difference and sum of quadrature amplitude of EPR state is then expressed as

$$
\left\langle\Delta^{2} \hat{X}_{-}^{\prime}\right\rangle=\left\langle\Delta^{2} \hat{Y}_{+}^{\prime}\right\rangle=2\left(1-L_{D}\right)(\mu-v)^{2}+2 L_{D},
$$

and the measured inseparability is

$$
I^{\prime}=2\left(1-L_{D}\right)(\mu-v)^{2}+2 L_{D} .
$$

Under the perfect detection efficiency $L_{D}=0$, we have $I^{\prime}=$ $2(\mu-v)^{2}$, which has the same value as $I_{s}$ in Eq. (3). Otherwise, the measured inseparability of the EPR source is $I^{\prime}>$ $2(\mu-v)^{2}$. So the entanglement is very fragile and vulnerable to losses, and the measured degree of entanglement will be degraded due to the existence of detection loss.

In addition to the loss-induced degradation on the measured entangled degree, there are other problems. First, the requirement of the availability of two BHDs with higher detection efficiency for measuring two different fields restricts the measurement technique to a certain extent. It is a challenge to measure entanglement between two fields of different types, if any one of them does not have the capability of BHD. For example, efficient detectors for $2-\mu \mathrm{m}$ optical field are not available yet. For hybrid entanglement between light and atom, the BHD technique is not available for atomic wave (see Sec. IV D for more). Second, $\hat{X}_{-}$and $\hat{Y}_{+}$cannot be measured simultaneously, that is, LO phases must be changed from 0 to $\pi / 2$ for two sets of measurement in order to respectively obtain the noise fluctuations of $\hat{X}_{-}$and $\hat{Y}_{+}$, from which the inseparability quantity $I_{s}$ can be deduced. Third, it is difficult to measure the broadband entanglement generated by a singlepass PA, whose noise reduction is in principle the same for the entire frequency band [22-24,29,30]. Since the response bandwidth of BHD is limited by the fixed value of product between the gain of electronic amplifier and gain bandwidth [see Fig. 1(b)], higher gain of amplifier is correlated to narrower gain bandwidth. Fourth, for multimode entangled state generated by pulse pumped PA, noise contributed by the mode mismatching between LOs and detected fields might be much larger than the vacuum noise due to the thermal nature of individual field of entanglement and phase asynchronization for different modes (see Sec. VB for more), which makes measured degree of pulsed entanglement smaller than what is anticipated [21,31].

\section{HOMODYNE DETECTION REALIZED BY THE COMBINATION OF HIGH-GAIN PSA AND A POWER DETECTOR}

The function of homodyne detection can also be realized by a high-gain PSA, whose strong pump serves as local oscillator $[19,20]$. Generally speaking, the PSA can be classified into two types. One is the degenerate PSA, in which the modes of the two inputs and outputs are the same; the other is nondegenerate PSA, in which the modes of two inputs and outputs are different. In this section, we will analyze the performance of such a type of homodyne detector in 


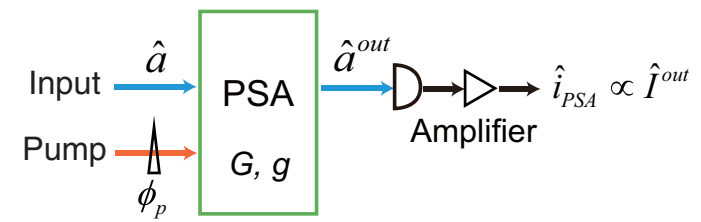

FIG. 2. The scheme of measuring the noise fluctuation of input field by using a degenerate high-gain phase-sensitive amplifier (PSA) followed by a power detector.

measuring noise reduction of optical fields when the PSAs are degenerate and nondegenerate, respectively.

\section{A. Degenerate phase-sensitive amplifier for measuring the noise fluctuation of an optical field}

Figure 2 shows the schematic diagram of measuring the noise fluctuation of input field $\hat{a}$ with a degenerate PSA. The output of PSA is directly measured by a power detector. The strong pump and weak input field are coherently combined via the nonlinear interaction in PSA. The relationship between the input and output fields of PSA is

$$
\hat{a}^{\text {out }}=G \hat{a}+g e^{i \varphi} \hat{a}^{\dagger},
$$

where $\hat{a}$ and $\hat{a}^{\text {out }}$ respectively represent the input and output fields, $G, g$ with $G^{2}-g^{2}=1$ are the amplitude gains of PSA and are mainly determined by the pump power, and $\varphi$ is the difference of phases between the pump and input fields. The intensity operator of the output field $\hat{a}^{\text {out }}$ is

$$
\begin{aligned}
\hat{I}^{\text {out }} & =\hat{a}^{\text {out }} \hat{a}^{\text {out }} \\
& =G^{2} \hat{a}^{\dagger} \hat{a}+g^{2} \hat{a} \hat{a}^{\dagger}+G g e^{i \varphi} \hat{a}^{\dagger} \hat{a}^{\dagger}+G g e^{-i \varphi} \hat{a} \hat{a} .
\end{aligned}
$$

The detector measures the average of the output intensity. If the input is in squeezed vacuum state [19], the average intensity at the output of PSA is written as

$$
\begin{aligned}
\left\langle\hat{I}^{\text {out }}\right\rangle= & \frac{(G+g)^{2}}{4}\left\langle\Delta^{2} \hat{X}^{2}(\varphi)\right\rangle \\
& +\frac{(G-g)^{2}}{4}\left\langle\Delta^{2} \hat{X}^{2}\left(\varphi+\frac{\pi}{2}\right)\right\rangle-\frac{1}{2} .
\end{aligned}
$$

Equation (11) shows the intensity is related to the noise of quadrature amplitude of input field. When the gain of PSA is very high $(G \sim g \gg 1)$ and the first term dominates, we then have the approximation

$$
\left\langle\hat{I}^{\text {out }}\right\rangle \approx g^{2}\left\langle\Delta^{2} \hat{X}(\varphi)\right\rangle .
$$

Equation (12) clearly illustrates that the noise of the quadrature amplitude of input field $\left\langle\Delta^{2} \hat{X}(\varphi)\right\rangle$ is amplified by the gain of PSA, and the phase difference $\varphi$ can be changed by changing the phase of pump. We note that traditional homodyne measurement gives directly the qradrature-phase amplitude $\hat{X}(\phi)$ while here from Eq. (4), one sees the average intensity of $\left\langle\hat{I}^{\text {out }}\right\rangle$ is proportional to the variance of $\hat{X}(\varphi)$, which is similar to the output of a spectral analyzer used in traditional homodyne measurement. Moreover, unlike the traditional homodyne measurement, the role of LO is replaced by the pump and the beam splitter is replaced by a PSA. The signal enhancement factor of the strong $\mathrm{LO}$ is equivalent to

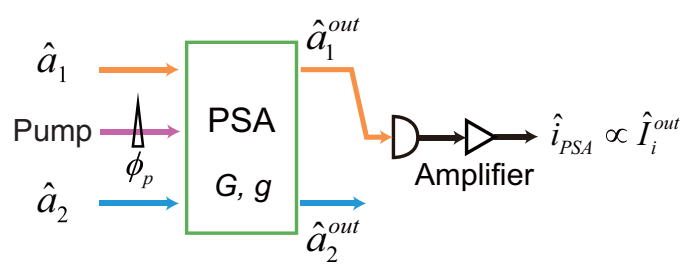

FIG. 3. Measuring the inseparability of two fields by using a nondegenerate high-gain phase-sensitive amplifier (PSA) followed by a power detector.

the gain of the PSA due to the pump. For example, for the $\chi^{(2)}$ crystal-based PSA, we have $\varphi=\phi_{p}-2 \phi_{\text {in }}$, where $\phi_{p}$ and $\phi_{\text {in }}$ are the phases of pump and input fields, respectively. The noises of two conjugated amplitudes of input, $\left\langle\Delta^{2} \hat{X}\right\rangle$ and $\left\langle\Delta^{2} \hat{Y}\right\rangle$, can be obtained by setting the phase $\varphi$ at 0 and $\pi$, at which the PSA is operated at the amplification and deamplification conditions, respectively. In order to characterize whether the noise is lower than vacuum, we need compare the noise of input field with the corresponding SNL,

$$
I_{\mathrm{SNL}}^{\text {out }}=g^{2},
$$

which is obtained by replacing the input field of PSA with vacuum. If the normalized intensity $\left\langle\hat{I}^{\text {out }}\right\rangle / I_{\mathrm{SNL}}^{\text {out }}$ is lower than 1 , the noise of input field $\left\langle\Delta^{2} \hat{X}(\varphi)\right\rangle$ is squeezed [19].

Although the noise of input field is amplified by the gain factor $g^{2}$, in practice, the gain of PSA cannot be infinitely large. To observe the output intensity of PSA, it is necessary to pass the photocurrent of detector through an electronic amplifier, whose output is expressed as

$$
\left\langle\hat{i}_{\mathrm{PSA}}\right\rangle=q^{2}\left\langle\hat{I}^{\mathrm{out}}\right\rangle=q^{2} g^{2}\left\langle\Delta^{2} \hat{X}(\varphi)\right\rangle,
$$

where $q^{2}$ is the gain of electrical amplifier. The comparison between Eqs. (14) and (4) shows that the parametric gain provided by strong pump of PSA functions as the LO of this kind of homodyne detection [19].

It is worth emphasizing that the approximation of Eq. (14) only holds when the gain PSA is very high. This is because in Eq. (11), $g$ should be large enough to ensure the first term dominates and the other terms are negligibly small. It is easy to make the second term small but it is not trivial to ensure the third term (originated from the commutation relation $\left[\hat{a}^{\text {out } \dagger}, \hat{a}^{\text {out }}\right]=1$ ) is negligible. For example, for the squeezed state input with $\left\langle\Delta^{2} \hat{X}(\varphi)\right\rangle=0.25$, the second term is more than a hundred times smaller than the first term when $g>3$. However, to make the third term 50 times smaller than the first term, $g>10$ is required.

\section{B. Nondegenerate phase-sensitive amplifier for measuring the inseparability of two optical fields}

Figure 3 shows the scheme for measuring the noise correlation between two fields by using a nondegenerate PSA, followed by a power detector. The modes of the two input fields of PSA, $\hat{a}_{1}, \hat{a}_{2}$, are nondegenerate, and the relation between the inputs and outputs of the PSA is

$$
\begin{aligned}
& \hat{a}_{1}^{\text {out }}=G \hat{a}_{1}+e^{i \varphi} g \hat{a}_{2}^{\dagger}, \\
& \hat{a}_{2}^{\text {out }}=G \hat{a}_{2}+e^{i \varphi} g \hat{a}_{1}^{\dagger},
\end{aligned}
$$


where $\hat{a}_{1}^{\text {out }}$ and $\hat{a}_{2}^{\text {out }}$ are the two output fields, and $\varphi$ is the difference between the pump phase and the sum of two input fields' phase [32]. Comparing Eq. (15) with Eqs. (9) and (5), one sees that the gain amplitude $G, g$ of the nondegenerate PSA not only amplifies the two inputs but also coherently combines $\hat{a}_{1}\left(\hat{a}_{2}\right)$ and $\hat{a}_{2}^{\dagger}\left(\hat{a}_{1}^{\dagger}\right)$, which is similar to the joint measurement of combining $\hat{X}_{1}$ and $\hat{X}_{2}$ with $g / G$ equivalent to $k$ in Eq. (5). So, it is reasonable to expect that one output of nondegenerate PSA can be used to characterize the entanglement between fields $\hat{a}_{1}$ and $\hat{a}_{2}$.

At one output of PA, says $\hat{a}_{1}^{\text {out }}$, the intensity operator is

$$
\hat{I}_{1}^{\text {out }}=\hat{a}_{1}^{\text {out } \dagger} \hat{a}_{1}^{\text {out }}=G^{2} \hat{a}_{1}^{\dagger} \hat{a}_{1}+g^{2} \hat{a}_{2} \hat{a}_{2}^{\dagger}+G g e^{i \varphi} \hat{a}_{2} \hat{a}_{1}+G g e^{-i \varphi} \hat{a}_{1}^{\dagger} \hat{a}_{2}^{\dagger} .
$$

The photocurrent of a power detector measures the average intensity of the field, $\left\langle\hat{I}_{1}^{\text {out }}\right\rangle$, and can be written as

$$
\begin{aligned}
\left\langle\hat{i}_{\mathrm{PSA}}\right\rangle= & q^{2}\left\langle\hat{I}_{1}^{\text {out }}\right\rangle=q^{2}\left(\frac{(G+g)^{2}}{16}\left\{\left\langle\Delta^{2}\left[\hat{X}_{1}(\varphi)+\hat{X}_{2}(\varphi)\right]\right\rangle+\left\langle\Delta^{2}\left[\hat{X}_{1}\left(\varphi+\frac{\pi}{2}\right)-\hat{X}_{2}\left(\varphi+\frac{\pi}{2}\right)\right]\right\rangle\right\}\right. \\
& \left.+\frac{(G-g)^{2}}{16}\left\{\left\langle\Delta^{2}\left[\hat{X}_{1}(\varphi)-\hat{X}_{2}(\varphi)\right]\right\rangle+\left\langle\Delta^{2}\left[\hat{X}_{1}\left(\varphi+\frac{\pi}{2}\right)+\hat{X}_{2}\left(\varphi+\frac{\pi}{2}\right)\right]\right\rangle\right\}-\frac{1}{2}\right)
\end{aligned}
$$

if there is no coherent component at each input to PSA or $\left\langle\hat{X}_{1}(\varphi)\right\rangle=0=\left\langle\hat{X}_{2}(\varphi)\right\rangle$, where $q^{2}$ is the gain of electronic amplifier. Similar to Eq. (11), when the gain of PSA is very high, the contribution of the first term in brace dominates and Eq. (17) can be approximated as

$$
\begin{aligned}
\left\langle\hat{i}_{\mathrm{PSA}}\right\rangle & =q^{2}\left\langle\hat{I}_{1}^{\text {out }}\right\rangle \\
& \approx q^{2} \frac{(G+g)^{2}}{16}\left[\left\langle\Delta^{2} \hat{X}_{+}(\varphi)\right\rangle+\left\langle\Delta^{2} \hat{X}_{-}\left(\varphi+\frac{\pi}{2}\right)\right\rangle\right] .
\end{aligned}
$$

To better understand Eq. (18), let us take the $\chi^{(2)}$-crystalbased nondegenerate PSA as an example. The phase difference is then $\varphi=\phi_{p}-\phi_{1}-\phi_{2}$, where $\phi_{p}$ is the phase of pump and $\phi_{1}$ and $\phi_{2}$ are the phases of two input fields. In the case of $\varphi=\pi$, PSA is operating at deamplification condition. Using the pump phase as a reference, we have $\phi_{1}=\phi_{2}=-\frac{\pi}{2}$, and the intensity of the photocurrent is written as

$$
\left\langle\hat{i}_{\mathrm{PSA}}\right\rangle=q^{2}\left\langle\hat{I}_{1}^{\text {out }}\right\rangle=q^{2} \frac{(G+g)^{2}}{16}\left[\left\langle\Delta^{2} \hat{X}_{-}\right\rangle+\left\langle\Delta^{2} \hat{Y}_{+}\right\rangle\right] .
$$

Normalizing $\left\langle\hat{i}_{\mathrm{PSA}}\right\rangle$ with the corresponding SNL obtained by placing two inputs at vacuum, $\left\langle\hat{i}_{\mathrm{PSA}}\right\rangle_{\mathrm{SNL}}=q^{2}\left\langle\hat{I}_{1}^{\mathrm{out}}\right\rangle_{\mathrm{SNL}}=$ $q^{2} g^{2}$, we find the ratio

$$
\frac{\left\langle\hat{i}_{\mathrm{PSA}}\right\rangle}{\left\langle\hat{i}_{\mathrm{PSA}}\right\rangle_{\mathrm{SNL}}}=I_{s} .
$$

The results indicate the intensity measured at one output of PSA can be used to characterize the inseparability of EPR source. On the other hand, when $\varphi=0$, i.e., the PSA operates at the amplification condition. If the fields to be measured are an entangled state generated from a PA described by Eq. (2), the combination of the PA there and the PSA here with $\varphi=0$ is equivalent to a PA with an overall gain of $G \mu-g \nu$ and $G v-g \mu$, which together generates an entangled state but with noise correlations of $\left\langle\Delta^{2} \hat{X}_{-}^{\text {out }}\right\rangle=\left\langle\Delta^{2} \hat{Y}_{+}^{\text {out }}\right\rangle=$ $(G-g)^{2}(\mu-v)^{2}[33]$.

Note that the intensity $\left\langle\hat{I}_{2}^{\text {out }}\right\rangle$ measured at the other output field $\hat{a}_{2}^{\text {out }}$ is similar to $\left\langle\hat{I}_{1}^{\text {out }}\right\rangle$ in Eq. (18). So the intensity measured at each output of PSA can be used to characterize the inseparability of the EPR source. Moreover, our analysis shows the nondegenerate PSA followed with a power detector can be used to characterize the inseparability of entanglement as long as the phase of PSA is properly locked and the gain of PSA is high enough, while the requirement of disentangling entanglement by PSA [20] is not necessary.

\section{Pros and cons of this type of homodyne detection}

Homodyne detections realized by PSA in Figs. 2 and 3 have the advantage of detection loss tolerance in measuring the noise reduction of the squeezed state and in checking the inseparability criterion. For example, for the PSA with $g \rightarrow \infty$, when the detection loss exists and is modeled as $L_{D}$, the output intensity is decreased to $\left\langle\hat{I}^{\text {out }}\right\rangle=\left(1-L_{D}\right)\left\langle\hat{I}^{\text {out }}\right\rangle$, and the corresponding SNL is also decreased to $\left\langle\hat{I}^{\text {out }}\right\rangle_{\mathrm{SNL}}=$ $\left(1-L_{D}\right)\left\langle\hat{I}_{1}^{\text {out }}\right\rangle_{\mathrm{SNL}}$. Hence, the normalized result of $\frac{\left\langle\hat{I}^{\text {out }}\right\rangle}{\left\langle\hat{I}^{\text {out }}\right\rangle_{\mathrm{SNL}}}=$ $\frac{\left\langle\hat{I}_{1}^{\text {out }}\right\rangle}{\left\langle\hat{I}_{1}^{\text {out }}\right\rangle_{\mathrm{SNL}}}$ is irrelevant to $L_{D}$. Moreover, unlike the traditional method which requires two BHDs and two sets of measurements, the inseparability of entanglement between two fields can be deduced from the intensity measured at only one output of nondegenerate PSA (see Fig. 3) and by only one measurement. This feature brings convenience in characterizing entanglement between two fields of different types.

However, there are bottlenecks which limit the practical application of this type of homodyne detections. First, the gain of PSA must be extremely high. Otherwise, the approximations in Eqs. (12) and (19) are not valid. In general, the achievable gain of PSA is mainly determined by the available pump power. The PSA with power gain higher than $20 \mathrm{~dB}$ $(g>10)$, at which this type of homodyne are experimentally demonstrated in Refs. [19,20], is usually difficult to realize. Second, the noise fluctuations of $\hat{X}_{-}=\hat{X}_{1}-\hat{X}_{2}$ and $\hat{Y}_{+}=$ $\hat{Y}_{1}+\hat{Y}_{2}$ of two entangled fields, which are the key in fulfilling the QIP tasks, such as quantum teleportation, quantum swapping, and quantum enhanced precision measurement, cannot be measured separately since the power detector in Figs. 2 and 3 lacks the ability to resolve phase information of detected fields. Third, the measurable bandwidth of the 


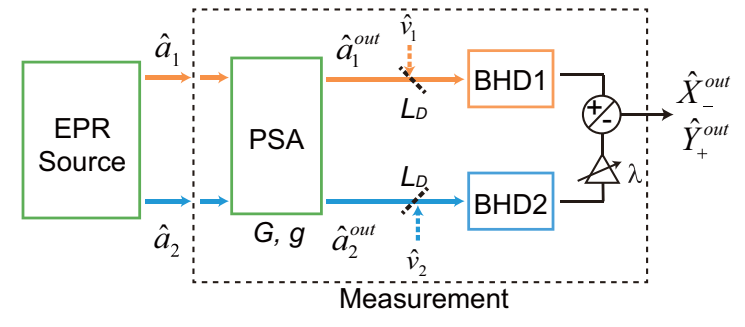

FIG. 4. Entanglement measured by assisting the traditional joint measurement of two balanced homodyne detection (BHD) with a nondegenerate phase-sensitive amplifier (PSA). The detection loss of each homodyne detection is modeled as a BS with reflectivity $L_{D}$. $\hat{v}_{1} / \hat{v}_{2}$ represents the vacuum field coupled into the detected fields.

quantum correlation of entanglement is relatively narrow. Although the coherent combination of two entangled field realized by the nonlinear coupling in PSA is much faster than that in traditional method (see Fig. 1), which is realized by taking the difference and sum of the photocurrents out of two BHDs with an electronic combiner, the response bandwidth of the power detector in Fig. 3 is usually very narrow for the following reason. From Eqs. (14) and (4), one sees that the optical amplification provided by the LO $\left(\left|\alpha_{L}\right|^{2}\right)$ in BHD can be much higher than that $\left(g^{2}\right)$ by the strong pump of PSA. So the gain of the electronic amplifier $q^{2}$ in Figs. 2 and 3 is usually much higher than that in Fig. 1(b), which severely limits the detection bandwidth due to gain-bandwidth relation of electronic amplifiers.

\section{OUR METHOD FOR MEASURING EPR ENTANGLEMENT WITH NONDEGENERATE PSA-ASSISTED BALANCED HOMODYNE DETECTORS}

Having reviewed two kinds of homodyne detection and their application in characterizing EPR entanglement, we study another method for measuring the entanglement by combining a nondegenerate PSA with balanced homodyne detections. During the measurement, two entangled fields are coupled into the PSA to reduce the influence of detection loss before extracting the phase information of the detected fields with BHDs. This method inherits the advantages of the measurement methods in Secs. II and III. In this section, we will first analyze two approaches of our method. One is to perform joint measurement by using two BHDs placed at each output of PSA. The other is to characterize the entanglement by using only one BHD at one output of PSA. Then we will study and compare the optimum operation condition for each approach by simulating the measurement results when the key parameters, such as gain of PSA and detection loss, are varied. At the end of this section, we will analyze the advantages and disadvantages of this method.

\section{A. Joint measurement performed by placing two BHDs at two outputs of PSA}

Figure 4 shows the approach by placing two BHDs at two outputs of a PSA and performing joint measurement. Two entangled fields $\hat{a}_{1}$ and $\hat{a}_{2}$ are coupled into the nondegenerate PSA with amplitude gain $G, g$, and the outputs $\hat{a}_{1}^{\text {out }}$ and $\hat{a}_{2}^{\text {out }}$ of
PSA are respectively measured by two BHDs. For simplicity, from here on, we focus on the measured quadrature components and drop the amplification term of each BHD provided by local and electronic amplifier, i.e., $\left|\alpha_{L}\right|^{2}=1$ and $q^{2}=1$. The relationship between the inputs and outputs of a PSA is same as in Eq. (15). When PSA is operating at deamplification condition $(\varphi=\pi)$, the input-output relation is

$$
\begin{aligned}
& \hat{a}_{1}^{\text {out }}=G \hat{a}_{1}-g \hat{a}_{2}^{\dagger}, \\
& \hat{a}_{2}^{\text {out }}=G \hat{a}_{2}-g \hat{a}_{1}^{\dagger} .
\end{aligned}
$$

When the LO phases of BHD1 and BHD2 are fixed at 0 or $\pi / 2$, the difference or sum of photocurrents of BHD1 and BHD2 measure the quantities $\hat{X}_{-}^{\text {out }} \equiv \hat{X}_{1}^{\text {out }}-\lambda \hat{X}_{2}^{\text {out }}$ and $\hat{Y}_{+}^{\text {out }} \equiv$ $\hat{Y}_{1}^{\text {out }}+\lambda \hat{Y}_{2}^{\text {out }}$, where $\lambda$ is the electronic gain of the current out of BHD2 to optimize the measurement results. Hence, the noise variances of joint measurement are related to the correlation of two entangled fields, $\hat{X}_{-}$and $\hat{Y}_{+}$, through the relation

$$
\begin{aligned}
& \left\langle\Delta^{2} \hat{X}_{-}^{\text {out }}\right\rangle=(G+\lambda g)^{2}\left\langle\Delta^{2}\left(\hat{X}_{1}-\frac{g+\lambda G}{G+\lambda g} \hat{X}_{2}\right)\right\rangle, \\
& \left\langle\Delta^{2} \hat{Y}_{+}^{\text {out }}\right\rangle=(G+\lambda g)^{2}\left\langle\Delta^{2}\left(\hat{Y}_{1}+\frac{g+\lambda G}{G+\lambda g} \hat{Y}_{2}\right)\right\rangle .
\end{aligned}
$$

The corresponding SNL for PSA-assisted joint measurement can be obtained by sending vacuum into both input ports of PSA. Hence, the SNL of the measurement scheme in Fig. 4 is expressed as

$$
\left\langle\Delta^{2} \hat{X}_{-}^{\text {out }}\right\rangle_{\mathrm{SNL}}=\left\langle\Delta^{2} \hat{Y}_{+}^{\text {out }}\right\rangle_{\mathrm{SNL}}=(G+\lambda g)^{2}+(g+\lambda G)^{2} .
$$

Normalizing Eq. (22) with the SNLs, we have

$$
\begin{aligned}
\left\langle\Delta^{2} \hat{X}_{-}^{\text {out }}\right\rangle_{N o r}= & \frac{\left\langle\Delta^{2} \hat{X}_{-}^{\text {out }}\right\rangle}{\left\langle\Delta^{2} \hat{X}_{-}^{\text {out }}\right\rangle_{\mathrm{SNL}}}=\frac{(G+\lambda g)^{2}}{(G+\lambda g)^{2}+(g+\lambda G)^{2}} \\
& \times\left\langle\Delta^{2}\left(\hat{X}_{1}-\frac{g+\lambda G}{G+\lambda g} \hat{X}_{2}\right)\right\rangle \\
\left\langle\Delta^{2} \hat{Y}_{+}^{\text {out }}\right\rangle_{N o r} & =\frac{\left\langle\Delta^{2} \hat{Y}_{+}^{\text {out }}\right\rangle}{\left\langle\Delta^{2} \hat{Y}_{+}^{\text {out }}\right\rangle_{\mathrm{SNL}}}=\frac{(G+\lambda g)^{2}}{(G+\lambda g)^{2}+(g+\lambda G)^{2}} \\
& \times\left\langle\Delta^{2}\left(\hat{Y}_{1}+\frac{g+\lambda G}{G+\lambda g} \hat{Y}_{2}\right)\right\rangle,
\end{aligned}
$$

and the inseparability

$$
I_{\mathrm{amp}}^{J M}=\left\langle\Delta^{2} \hat{X}_{-}^{\text {out }}\right\rangle_{N o r}+\left\langle\Delta^{2} \hat{Y}_{+}^{\text {out }}\right\rangle_{N o r} .
$$

where the superscript $J M$ stands for joint measurement. For the case of $\lambda=1\left(\frac{g+\lambda G}{G+\lambda g}=1\right)$, we have the noise reduction due to entanglement correlation,

$$
\begin{aligned}
& \left\langle\Delta^{2} \hat{X}_{-}^{\text {out }}\right\rangle_{N o r}=\left\langle\Delta^{2} \hat{X}_{-}\right\rangle_{s}=(\mu-v)^{2}, \\
& \left\langle\Delta^{2} \hat{Y}_{+}^{\text {out }}\right\rangle_{N o r}=\left\langle\Delta^{2} \hat{Y}_{+}\right\rangle_{s}=(\mu-v)^{2},
\end{aligned}
$$

and the inseparability in Eq. (25) reach the minimum $I_{s}$ [see Eq. (3)] for arbitrary value of $g$. So the optimum results of Eqs. (24) and (25) are always the same as those measured by the traditional method under perfect detection efficiency. Moreover, the analysis indicates traditional method 


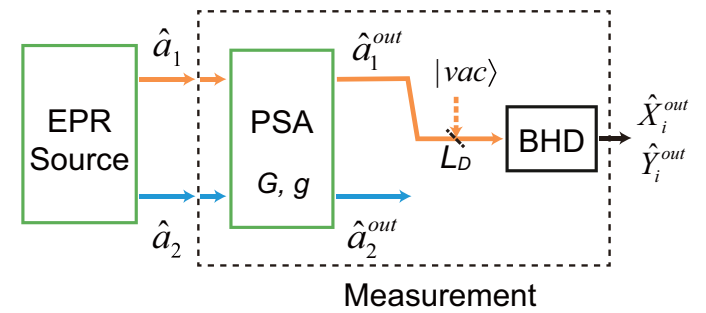

FIG. 5. Entanglement measurement performed by placing one balanced homodyne detection (BHD) at one output of nondegenerate phase-sensitive amplifier (PSA). The detection loss of BHD is modeled as a BS with reflectivity $L_{D}$.

in Sec. II B is just a special case of PSA-assisted joint measurement with the gain of PSA set to $1(G=1)$. This PSA-assisted scheme provides a parametric gain of $g>0$ for amplifying the noise correlation of entangled fields and shot noise level of the measurement with the factor $(G+\lambda g)^{2}$. So, it is straightforward to predict that the ability of detection loss tolerance for measurement scheme in Fig. 4 improves with the increase of $g$. The detailed information of how the measurement results depend on the gain of PSA and loss of detection will be given in Sec. IV C.

\section{B. Homodyne detection performed at one output of the PSA}

Similar to the situation in Secs. III B and III C, that is, that the intensity directly measured at one output of nondegenerate PSA can be used to characterize the inseparability of entanglement, let us examine what will come out if we replace the power detector in Fig. 3 with a BHD, as shown in Fig. 5.

Again, we assume the nondegenerate PSA in Fig. 5 is working at the deamplification condition for measuring the entanglement produced from the scheme in Fig. 1(a). According to the relationship between the inputs and outputs in Eq. (21), the variances of quadrature amplitudes $\hat{X}_{1}^{\text {out }}$ or $\hat{Y}_{1}^{\text {out }}$ at one output field, say, $\hat{a}_{1}^{\text {out }}$, are related to the correlation of the source, $\hat{X}_{-}$and $\hat{Y}_{+}$, through the relation:

$$
\begin{aligned}
\left\langle\Delta^{2} \hat{X}_{1}^{\text {out }}\right\rangle & =G^{2}\left\langle\Delta^{2}\left(\hat{X}_{1}-\frac{g}{G} \hat{X}_{2}\right)\right\rangle, \\
\left\langle\Delta^{2} \hat{Y}_{1}^{\text {out }}\right\rangle & =G^{2}\left\langle\Delta^{2}\left(\hat{Y}_{1}+\frac{g}{G} \hat{Y}_{2}\right)\right\rangle,
\end{aligned}
$$

which are obtained by locking the LO of BHD at 0 and $\pi / 2$, respectively, indicating that the noise variances of $\left\langle\Delta^{2}\left(\hat{X}_{1}-\frac{g}{G} \hat{X}_{2}\right)\right\rangle$ and $\left\langle\Delta^{2}\left(\hat{Y}_{1}+\frac{g}{G} \hat{Y}_{2}\right)\right\rangle$ are amplified with the factor $G^{2}$. On the other hand, the corresponding SNLs can be obtained by sending vacuum into both input ports of PSA. Hence, the SNLs of the measurement scheme are given by

$$
\left\langle\Delta^{2} \hat{X}_{1}^{\text {out }}\right\rangle_{\mathrm{SNL}}=\left\langle\Delta^{2} \hat{Y}_{1}^{\text {out }}\right\rangle_{\mathrm{SNL}}=G^{2}+g^{2} .
$$

Normalizing Eq. (27) with the SNLs in Eq. (28), we have

$$
\begin{aligned}
& \left\langle\Delta^{2} \hat{X}_{1}^{\text {out }}\right\rangle_{\text {Nor }}=\frac{\left\langle\Delta^{2} \hat{X}_{1}^{\text {out }}\right\rangle}{\left\langle\Delta^{2} \hat{X}_{1}^{\text {out }}\right\rangle_{\mathrm{SNL}}}=\frac{G^{2}}{G^{2}+g^{2}}\left\langle\Delta^{2}\left(\hat{X}_{1}-\frac{g}{G} \hat{X}_{2}\right)\right\rangle, \\
& \left\langle\Delta^{2} \hat{Y}_{1}^{\text {out }}\right\rangle_{N o r}=\frac{\left\langle\Delta^{2} \hat{Y}_{1}^{\text {out }}\right\rangle}{\left\langle\Delta^{2} \hat{Y}_{1}^{\text {out }}\right\rangle_{\mathrm{SNL}}}=\frac{G^{2}}{G^{2}+g^{2}}\left\langle\Delta^{2}\left(\hat{Y}_{1}+\frac{g}{G} \hat{Y}_{2}\right)\right\rangle,
\end{aligned}
$$

and the inseparability

$$
I_{\mathrm{amp}}^{(1)}=\left\langle\Delta^{2} \hat{X}_{1}^{\text {out }}\right\rangle_{N o r}+\left\langle\Delta^{2} \hat{Y}_{1}^{\text {out }}\right\rangle_{N o r},
$$

where the superscript "(1)" denotes the output field of $\hat{a}_{1}^{\text {out }}$. When the gain of PSA is very high, i.e., $g / G \rightarrow 1$ (or $g \rightarrow$ $\infty)$, for the EPR source in Fig. 1(a), we have the noise reduction

$$
\begin{aligned}
\left\langle\Delta^{2} \hat{X}_{1}^{\text {out }}\right\rangle_{N o r} & =\left\langle\Delta^{2} \hat{X}_{-}\right\rangle_{s}=(\mu-v)^{2}, \\
\left\langle\Delta^{2} \hat{Y}_{1}^{\text {out }}\right\rangle_{N o r} & =\left\langle\Delta^{2} \hat{Y}_{+}\right\rangle_{s}=(\mu-v)^{2},
\end{aligned}
$$

and inseparability $I_{\text {amp }}^{(1)} \rightarrow 2(\mu-v)^{2}$, which are exactly the same as the fluctuations in Eq. (2) and inseparability $I_{s}$ in Eq. (3). Note that if the BHD is placed at the output of $\hat{a}_{2}^{\text {out }}$, the results are similar to those measured at $\hat{a}_{1}^{\text {out }}$ output, except respectively replacing $g$ and $G$ in Eq. (29) with $G$ and $g$. So, when $g / G \rightarrow 1$ (or $g \rightarrow \infty$ ), similar to Eq. (31), we have $\left\langle\Delta^{2} \hat{X}_{2}^{\text {out }}\right\rangle_{\text {Nor }}=\left\langle\Delta^{2} \hat{X}_{-}\right\rangle_{s}=(\mu-v)^{2},\left\langle\Delta^{2} \hat{Y}_{2}^{\text {out }}\right\rangle_{\text {Nor }}=$ $\left\langle\Delta^{2} \hat{Y}_{+}\right\rangle_{s}=(\mu-v)^{2}$. Hence, we can measure $\left\langle\Delta^{2} \hat{X}_{-}\right\rangle_{s}$ at output $\hat{a}_{1}^{\text {out }}$ and in the same time $\left\langle\Delta^{2} \hat{Y}_{+}\right\rangle_{s}$ at output $\hat{a}_{2}^{\text {out }}$, obtaining the inseparability quantity $I_{s}$ with one set of measurement.

It is worth noting that the coherent combination of two entangled fields realized by nondegenerate PSA is different from that realized by subtracting or adding up the photocurrents of two BHDs, which is a linear combination of the quadrature amplitudes of $\hat{X}_{1}(\phi)$ and $\hat{X}_{2}(\phi)$. From Eq. (21), one sees that PSA can coherently combine one input field with the conjugate of the other input field. So, in contrast to the traditional method, in which the measurement results of noise variance highly depend on LO phase of each BHD [9], the noise variance of quadrature amplitude measured by the BHD at one output of PSA is irrelevant to LO phase [34]. For example, for the entangled state produced by the source in Fig. 1(a), we have the normalized noise fluctuation

$$
\left\langle\Delta^{2} \hat{X}_{1}^{\text {out }}(\phi)\right\rangle_{\text {Nor }}=\frac{(\mu G-v g)^{2}+(\mu g-v G)^{2}}{\left(G^{2}+g^{2}\right)},
$$

where $\phi$ is the LO phase of BHD. Equation (32) clearly indicates that the variance of quadrature amplitude at arbitrary angle $\phi,\left\langle\hat{X}_{1}^{\text {out }}(\phi)\right\rangle_{\text {Nor }}$, depends on the gains of both the entangled source and PSA but does not vary with the LO phase of BHD. This is because of the destructive quantum interference effect in PSA [35]. However, to extract information carried by the entangled fields, correctly locking the phase is still the key to measure the encoded information. For example, when one field of the entangled source is encoded with the information of both weak amplitude and phase modulations, we need to decode the information by respectively measuring the quadrature amplitudes $\hat{X}_{1}^{\text {out }}$ and $\hat{Y}_{2}^{\text {out }}$ with the LO phase locked at 0 and $\pi / 2$ [34]. Notice that $\hat{X}_{1}^{\text {out }}$ and $\hat{Y}_{2}^{\text {out }}$ are measured at different output ports and can be done in the same time, thus achieving simultaneous measurement of amplitude and phase $[34,36]$.

Unlike Eqs. (24) and (25), whose optimum values are irrelevant to the gain of PSA, Eqs. (29)-(32) show that the optimized measurement of entanglement requires the condition of $g \rightarrow \infty$. In practice, it is impossible to achieve $g \rightarrow \infty$ since the pump power of PSA cannot be infinitely high. In order to understand how the gain of PSA affects the performance of the scheme in Fig. 5, we analyze the gain dependence 


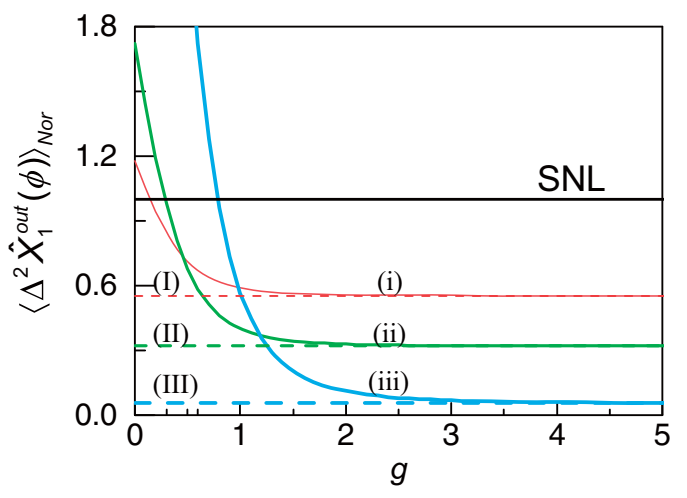

FIG. 6. The value of $\left\langle\Delta^{2} \hat{X}_{1}^{\text {out }}(\phi)\right\rangle_{N o r}$ measured by one balanced homodyne detection vs the gain amplitude $g$ of PSA when the noise reduction of entangled source is fixed at different levels. The dashed lines labeled as (I), (II), and (III) represent the noise reduction of the source $\left\langle\Delta^{2} \hat{X}_{-}\right\rangle_{s}=\left\langle\Delta^{2} \hat{Y}_{+}\right\rangle_{s}$ at the levels of $0.554,0.321$, and 0.056 , which can be viewed as the result achievable with perfect detection. The solid curves labeled as (i), (ii), and (iii) represent the measured results for the cases of (I), (II), and (III), respectively. The solid black line denotes the shot noise levels (SNL).

of measurement results by taking the entangled source in Fig. 1(a) as an example. Since $\left\langle\Delta^{2} \hat{X}_{1}^{\text {out }}\right\rangle_{N o r}=\left\langle\Delta^{2} \hat{Y}_{1}^{\text {out }}\right\rangle_{N o r}=$ $\left\langle\Delta^{2} \hat{X}_{1}^{\text {out }}(\phi)\right\rangle_{N o r}$, we will calculate $\left\langle\Delta^{2} \hat{X}_{1}^{\text {out }}(\phi)\right\rangle_{N o r}$ as a function of $g$ when the noise correlation $\left\langle\Delta^{2} \hat{X}_{-}\right\rangle_{s}$ (or $\left.\left\langle\Delta^{2} \hat{Y}_{+}\right\rangle_{s}\right)$ of two entangled fields directly out of the source is fixed at different levels, as shown in Fig. 6. In the calculation, the given values $\left\langle\Delta^{2} \hat{X}_{-}\right\rangle_{s}$ of EPR source or the result with perfect detection are $0.554,0.321$, and 0.056 [dashed lines (I), (II), and (III) in Fig. 6], which correspond to the squeezing degrees of 2.6, 4.9, and $12.5 \mathrm{~dB}$, respectively. Although the noise of a real entanglement source is determined by the PA with certain amounts of gain and internal loss, for the sake of brevity, we neglect the internal loss and obtain the noise of entangled fields by substituting the gain values of source $v=$ $0.3,0.6,2$ into Eq. (2). The value $\left\langle\Delta^{2} \hat{X}_{1}^{\text {out }}(\phi)\right\rangle_{\text {Nor }}$ measured with the PSA-assisted scheme is obtained by substituting the different gain values of PSA and PA into Eq. (32). In Fig. 6, the solid curves labeled as (i), (ii), and (iii) represent the measured value $\left\langle\Delta^{2} \hat{X}_{1}^{\text {out }}(\phi)\right\rangle_{N o r}$ for the EPR source with ideal results labeled as (I), (II), and (III), respectively. It is clear that in each case, the deviation between the measured value $\left\langle\Delta^{2} \hat{X}_{1}^{\text {out }}(\phi)\right\rangle_{\text {Nor }}$ and ideally measurable value $\left\langle\Delta^{2} \hat{X}_{-}\right\rangle_{s}$ (dashed line) decreases with the increase of $g$. When $g$ is low, the value of $\left\langle\Delta^{2} \hat{X}_{1}^{\text {out }}(\phi)\right\rangle_{N o r}$ is higher than the SNL of 1 . In particular, when $g=0$, we have $\hat{X}_{1}^{\text {out }}=\hat{X}_{1}$. In this case, the value of $\left\langle\Delta^{2} \hat{X}_{1}^{\text {out }}(\phi)\right\rangle_{N o r}$ is always higher than the normalized SNL of 1 and increases with the gain of the source because each individual field $\left(\hat{X}_{1}\right)$ of the entangled source is in a thermal state. When $g$ is higher than a certain level, the value of $\left\langle\Delta^{2} \hat{X}_{1}^{\text {out }}(\phi)\right\rangle_{N o r}$ starts to become lower than 1 . In each case, when $g$ is greater than 3, which corresponds to PSA having a power gain of $G^{2}=10,\left\langle\Delta^{2} \hat{X}_{1}^{\text {out }}\right\rangle_{N o r}$ becomes very close to the noise reduction of the source $\left\langle\Delta^{2} \hat{X}_{-}\right\rangle_{s}$. In real experiment, it is easy to achieve the gain level of $G^{2}=10$. Therefore, comparing with the scheme in Sec. III, the requirement to the gain of PSA is greatly relaxed by conducting measurement with the combination of PSA and BHD.

\section{Influence of detection loss upon the performance of this measurement method}

Having explained the working principle of this method, we are ready to analyze the influence of detection loss. Let us first investigate the performance of PSA-assisted joint measurement scheme in Fig. 4. For the sake of convenience, we assume that the electronic gain is $\lambda=1$ and the detection losses of the two BHDs are both $L_{D}$. With the presence of detection losses, the joint measurement quantities in Eq. (25) is modified to

$$
\begin{aligned}
\left\langle\Delta^{2} \hat{X}_{-}^{\text {out }}\right\rangle & =\left\langle\Delta^{2}\left(\hat{X}_{1}^{\text {out }^{\prime}}-\hat{X}_{2}^{\text {out }}\right)\right\rangle \\
& =\left(1-L_{D}\right)\left\langle\Delta^{2}\left(\hat{X}_{1}^{\text {out }}-\hat{X}_{2}^{\text {out }}\right)\right\rangle+2 L_{D}, \\
\left\langle\Delta^{2} \hat{Y}_{+}^{\text {out }}\right\rangle & =\left\langle\Delta^{2}\left(\hat{Y}_{1}^{\text {out }{ }^{\prime}}+\hat{Y}_{2}^{\text {out }}\right)\right\rangle \\
& =\left(1-L_{D}\right)\left\langle\Delta^{2}\left(\hat{Y}_{1}^{\text {out }}+\hat{Y}_{2}^{\text {out }}\right)\right\rangle+2 L_{D},
\end{aligned}
$$

and the corresponding SNL is

$$
\left\langle\Delta^{2} \hat{X}_{-}^{\text {out }^{\prime}}\right\rangle_{\mathrm{SNL}}=\left\langle\Delta^{2} \hat{Y}_{+}^{\text {out }^{\prime}}\right\rangle_{\mathrm{SNL}}=2\left(1-L_{D}\right)(G+g)^{2}+2 L_{D} .
$$

It is straightforward to show that the second term of detection loss $2 L_{D}$ in Eqs. (33) and (34) can be dropped if the gain of PSA $g$ is large. When the gain of PSA satisfies the condition

$$
\left(1-L_{D}\right)(G+g)^{2} \gg 1,
$$

the noise reduction and inseparability measured by joint measurement are modified to

$$
\begin{aligned}
\left\langle\Delta^{2} \hat{X}_{-}^{\text {out }}\right\rangle_{N o r} & =\left\langle\Delta^{2} \hat{X}_{-}\right\rangle_{s}=(\mu-v)^{2}, \\
\left\langle\Delta^{2} \hat{Y}_{+}^{\text {out }}{ }^{\prime}\right\rangle_{N o r} & =\left\langle\Delta^{2} \hat{Y}_{+}\right\rangle_{s}=(\mu-v)^{2}, \\
I_{\text {amp }}^{J M^{\prime}} & =I_{s},
\end{aligned}
$$

which means we can measure entanglement with results immune to detection loss as long as the gain of PSA is high enough. For example, for the entangled fields generated by the source in Fig. 1(a), the measured noise reduction and inseparability can be written as

$$
\begin{aligned}
\left\langle\Delta^{2} \hat{X}_{-}^{\text {out }}{ }^{\prime}\right\rangle_{N o r} & =\left\langle\Delta^{2} \hat{Y}_{+}^{\text {out }{ }^{\prime}}\right\rangle_{N o r} \\
& =\frac{\left(1-L_{D}\right)(G+g)^{2}(\mu-v)^{2}+L_{D}}{\left(1-L_{D}\right)(G+g)^{2}+L_{D}}, \\
I_{\text {amp }}^{J M^{\prime}}= & \frac{2\left(1-L_{D}\right)(G+g)^{2}(\mu-v)^{2}+2 L_{D}}{\left(1-L_{D}\right)(G+g)^{2}+L_{D}} .
\end{aligned}
$$

When $\left(1-L_{D}\right)(G+g)^{2} \gg 1$ holds, the measured values $\left\langle\Delta^{2} \hat{X}_{-}^{\text {out }^{\prime}}\right\rangle_{N o r}=\left\langle\Delta^{2} \hat{Y}_{+}^{\text {out }^{\prime}}\right\rangle_{N o r}$ and $I_{\text {amp }}^{J M^{\prime}}$ approach to $(\mu-v)^{2}$ and $2(\mu-v)^{2}$, which are the same as $\left\langle\Delta^{2} \hat{X}_{-}\right\rangle_{s}=\left\langle\Delta^{2} \hat{Y}_{+}\right\rangle_{s}$ and $I_{s}$ directly out of the source [see Eqs. (2) and (3)].

To better illustrate influence of detection loss on the scheme in Fig. 4, we simulate the results $I_{\mathrm{amp}}^{J M^{\prime}}$ when the gain of PSA is fixed at different levels. The calculation is carried out by using Eq. (38), in which the inseparability of the source $I_{s}=0.112$ is obtained by setting $v=2$. Figure 7 (a) plots $I_{\text {amp }}^{J M^{\prime}}$ as a function of detection loss when the gain of PSA is respectively fixed at $g=0,2,3,5$. We note that the case of $g=0$ is equivalent to the traditional method in Fig. 1, and best estimation of inseparability is obtained under the ideal 
(a)

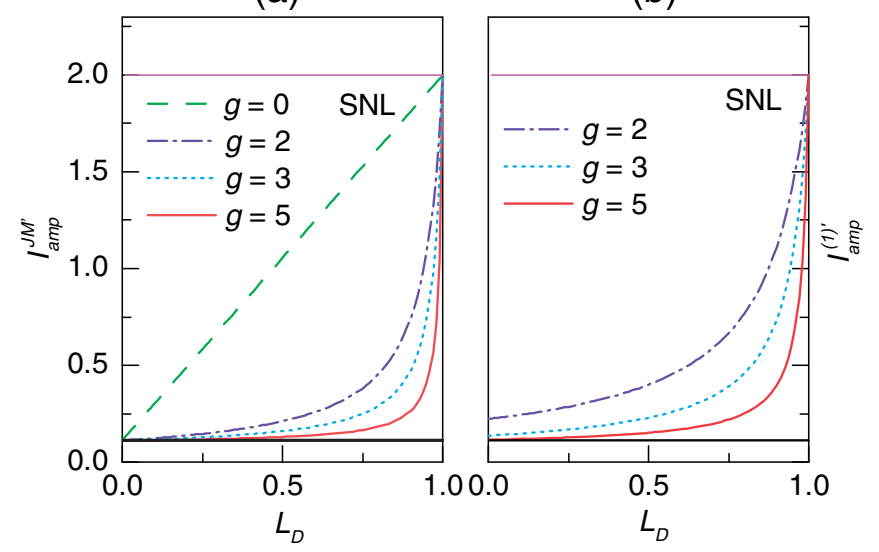

FIG. 7. (a) Jointly measured inseparability $I_{\text {amp }}^{J M^{\prime}}$ as a function the detection loss $L_{D}$ when the gain amplitude $g$ of PSA in Fig. 4 is fixed at $g=0,2,3,5$, respectively. (b) Inseparability $I_{\text {amp }}^{(1)}$ ' measured by the individual BHD as a function of $L_{D}$ when the gain amplitude $g$ of PSA in Fig. 5 is fixed at $g=2,3,5$, respectively. In the calculation, the inseparability of the source is $I_{s}=0.112$. The dashed line $(g=0)$ corresponds to the results of traditional method, and the black solid line represents the best estimation of inseparability obtained under the ideal detection condition of $L_{D}=0$.

detection condition of $L_{D}=0$. It is clear that for the case of $L_{D}=0$, the measured inseparability $I_{\mathrm{amp}}^{J M^{\prime}}$ always equal to $I_{s}$ and is irrelevant to gain of PSA. When $g$ is fixed, the general trend of $I_{\mathrm{amp}}^{J M^{\prime}}$ is to increase with $L_{D}$; however, the rising slope decreases with the increase $g$. In this case, the rising slope of $I_{\text {amp }}^{J M^{\prime}}$ versus $L_{D}$ is the highest. When $L_{D}$ increases from 0 to $0.6, I_{\mathrm{amp}}^{J M^{\prime}}$ increases from 0.112 to 1.2 . Whereas for the case of $g=5$, corresponding to PSA with power gain of about 26, the rising slope of $I_{\text {amp }}^{J M}$ is very low. Even if the detection loss is 0.6 , we still have $I_{\text {amp }}^{J M^{\prime}}=0.13$, which is only slightly higher than that obtained with perfect detection. The results indicate the role of PSA in Fig. 4 is to mitigate the influence of detection loss on entanglement measurement.

Let us now analyze the scheme of measuring entanglement at only one output of PSA with one BHD when the nonideal efficiency of BHD is taken into account. In this case, Eq. (27), which describes the noise levels measured at $\hat{a}_{1}^{\text {out }}$ output of PSA-assisted scheme, has the modified form:

$$
\begin{aligned}
\left\langle\Delta^{2} \hat{X}_{1}^{\text {out }}\right\rangle & =\left(1-L_{D}\right)\left\langle\Delta^{2} \hat{X}_{1}^{\text {out }}\right\rangle+L_{D} \\
& =\left(1-L_{D}\right) G^{2}\left\langle\Delta^{2}\left(\hat{X}_{1}-\frac{g}{G} \hat{X}_{2}\right)\right\rangle+L_{D} \\
\left\langle\Delta^{2} \hat{Y}_{1}^{\text {out }}\right\rangle & =\left(1-L_{D}\right)\left\langle\Delta^{2} \hat{Y}_{1}^{\text {out }}\right\rangle+L_{D} \\
& =\left(1-L_{D}\right) G^{2}\left\langle\Delta^{2}\left(\hat{Y}_{1}+\frac{g}{G} \hat{Y}_{2}\right)\right\rangle+L_{D}
\end{aligned}
$$

Normalizing Eq. (39) with the corresponding shot noise level of $\left\langle\Delta^{2} \hat{X}_{1}^{\text {out }^{\prime}}\right\rangle_{\mathrm{SNL}}=\left\langle\Delta^{2} \hat{Y}_{1}^{\text {out }^{\prime}}\right\rangle_{\mathrm{SNL}}=\left(1-L_{D}\right)\left(G^{2}+g^{2}\right)+L_{D}$, Eqs. (29) and (30) are modified as

$$
\left\langle\Delta^{2} \hat{X}_{1}^{\text {out }^{\prime}}\right\rangle_{N o r}=\frac{\left(1-L_{D}\right) G^{2}\left\langle\Delta^{2} \hat{X}_{-}\right\rangle+L_{D}}{\left(1-L_{D}\right)\left(G^{2}+g^{2}\right)+L_{D}},
$$

$$
\begin{aligned}
\left\langle\Delta^{2} \hat{Y}_{1}^{\text {out }}\right\rangle_{N o r} & =\frac{\left(1-L_{D}\right) G^{2}\left\langle\Delta^{2} \hat{Y}_{+}\right\rangle+L_{D}}{\left(1-L_{D}\right)\left(G^{2}+g^{2}\right)+L_{D}}, \\
I_{\text {amp }}^{(1)^{\prime}} & =\frac{\left(1-L_{D}\right) G^{2}\left[\left\langle\Delta^{2} \hat{X}_{-}\right\rangle+\left\langle\Delta^{2} \hat{Y}_{+}\right\rangle\right]+2 L_{D}}{\left(1-L_{D}\right)\left(G^{2}+g^{2}\right)+L_{D}} .
\end{aligned}
$$

When the relation

$$
\left(1-L_{D}\right) G^{2} \gg 1
$$

holds, the second term of loss $L_{D}$ in both numerator and denominator of Eq. (40) can be dropped. In this case, we have

$$
\begin{aligned}
\left\langle\Delta^{2} \hat{X}_{1}^{\text {out }}\right\rangle_{N o r} & \approx\left\langle\Delta^{2} \hat{X}_{-}\right\rangle_{s}, \\
\left\langle\Delta^{2} \hat{Y}_{1}^{\text {out }}\right\rangle_{N o r} & \approx\left\langle\Delta^{2} \hat{Y}_{+}\right\rangle_{s}, \\
I_{\text {amp }}^{(1) '} & \approx I_{s} .
\end{aligned}
$$

Since the measurement obtained by placing the BHD at each output of high gain PSA is the same, the expressions in Eq. (42) indicate that when the gain of PSA is large enough, we can measure the entanglement of EPR source with the measurement results immune to detection loss. Taking the entangled source in Fig. 1(a) as an example, Eq. (40) can be rewritten as

$$
\begin{aligned}
\left\langle\Delta^{2} \hat{X}_{1}^{\text {out }}\right\rangle_{N o r} & =\left\langle\Delta^{2} \hat{Y}_{1}^{\text {out }{ }^{\prime}}\right\rangle_{N o r} \\
& =\frac{\left(1-L_{D}\right)\left[(\mu G-v g)^{2}+(\mu g-v G)^{2}\right]+L_{D}}{\left(1-L_{D}\right)\left(G^{2}+g^{2}\right)+L_{D}}, \\
I_{\mathrm{amp}}^{(1)^{\prime}} & =\frac{2\left(1-L_{D}\right)\left[(\mu G-v g)^{2}+(\mu g-v G)^{2}\right]+2 L_{D}}{\left(1-L_{D}\right)\left(G^{2}+g^{2}\right)+L_{D}} .
\end{aligned}
$$

When $\left(1-L_{D}\right) G^{2} \gg 1$ holds, the measured inseparability $I_{\text {amp }}^{(1)}$ ' approaches to $2(\mu-v)^{2}$, which is the ideal measurement result of $I_{s}$ [see Eq. (3)].

We have shown in Sec. IV B that measuring entanglement with one BHD only works for the PSA with high gain, so we will only analyze the influence of loss on the measurement when the gain $g$ of the PSA in Fig. 5 is relatively high. We carry out the calculation by using Eq. (38), in which the entangled source with $I_{s}=0.112$ is the same as in Fig. 7(a). Figure 7(b) shows $I_{\text {amp }}^{(1)}$ ' as a function of detection loss when the gain of PSA is respectively fixed at $g=2,3,5$. One sees that in each case, $I_{\text {amp }}^{(1)}$ ' slightly increases with $L_{D}$. Moreover, the rising slope of $I_{\mathrm{amp}}^{(1)}$ ' decreases with the increase of $g$. Note that even if the detection efficiency if perfect $\left(L_{D}=0\right), I_{\mathrm{amp}}^{(1)^{\prime}}$ slightly deviates from the thick solid line, representing the deviation between $I_{\mathrm{amp}}^{(1)}$ ' and $I_{S}$ decreases with the increase of $g$. The results indicate that in order to measure entanglement with only one BHD, the gain of PSA in Fig. 5 should be high enough even if the detection efficiency is high. Moreover, comparing Fig. 7(b) with Fig. 7(a), we find that if the gain of PSA is the same, the deviation between $I_{\mathrm{amp}}^{J M^{\prime}}$ and $I_{s}$ is always smaller than that between $I_{\mathrm{amp}}^{(1)}{ }^{\prime}$ and $I_{s}$. So, the loss tolerance feature for the approach in Fig. 4 is better than that in Fig. 5. In the treatment of losses in this paper, we have not considered the internal loss of the parametric amplifier. This type of loss 
is complicated and has some detrimental effects that cannot be overcome by the current scheme [35].

\section{Pros and cons of our measurement method}

The results in Fig. 7 clearly shows that compared to the traditional method [see the curve for $g=0$ (or $G=1$ ) in Fig. 7(a)], both approaches of this method have the advantage of detection loss tolerance. If the measurement results are obtained from the approach of joint measurement of two BHDs, the ability of loss tolerance increases with $g$, and this method is always better than the traditional one as long as the gain of PSA is bigger than 1. If only one output of PSA is measured by a BHD, the advantage of our method can be realized only when the gain (or pump power) of PSA is high. Hence, the scheme with only one BHD in Fig. 5 seems to be less attractive. However, an interesting feature for the approach in Fig. 5 is that only one BHD is required, which should be useful for characterizing or measuring entanglement between two different types of waves where one of the waves is hard to measure. For example, for the hybrid entanglement between atom and light, it is impossible to use the traditional method because BHD of atomic wave is not available, but we can use an atomic Raman amplifier, which is essentially a parametric amplifier for light and atomic excitation waves [16-18], to combine these two different types of waves. The quantum correlation between atom and light can then be characterized by only performing homodyne detection on the optical field.

If we are able to perform BHD at both output ports, we can then measure $\hat{X}_{-}$at one port and $\hat{Y}_{+}$at the other, achieving simultaneous measurement of $\hat{X}_{-}$and $\hat{Y}_{+}$and obtaining $I_{s}$ with one set of measurement. This is somewhat similar to the method discussed in Sec. III and is thus more advantageous than the traditional method discussed in Sec. II. Of course, our method can measure $\hat{X}_{-}$and $\hat{Y}_{+}$separately, which is also more advantageous than the method discussed in Sec. III, where measurement of $\hat{X}_{-}$and $\hat{Y}_{+}$cannot be separated in the output.

Furthermore, even if we only have one output port with BHD capability (e.g., atomic Raman amplifier), because the PSA's output is tolerant to vacuum noise at high gain, we can split this output into two by a beam splitter without worrying about the vacuum noise introduced through the unused port of the beam splitter and perform separate BHD to obtain $\hat{X}_{-}$and $\hat{Y}_{+}$independently at the same time.

Another potential advantage provided by this method is that the response bandwidth of detection can be greatly improved. On the one hand, the coherent combination of two entangled fields realized by the nonlinear coupling in high gain PSA is much fast than that the electronic combiner used in traditional method. On the other hand, the noises of entangled state measured by this method have experienced two kinds of amplification. In addition to the amplification provided by the LO of BHD, the noises are amplified by PSA with high gain. As shown in Eqs. (13), (23), and (28), the SNL of the our measurement method is lifted by the high-gain PSA. Therefore, we achieve by an optical method the goal of effectively amplifying a weak input signal to a level that is otherwise buried in the classical noise such as detector's dark current so that thermal electronic noise of current amplifiers and gain of electronic amplifier can be relaxed, which will result in an increased gain bandwidth in measurement.

Despite these advantages, it is worth noting that this method can overcome the problem of only detection loss. For the losses occurred inside the entangled source of PA and in coupling the entangled source into the PSA, on the other hand, the usage of PSA is not useful because these losses have negative effects on the entangled degree, which is equivalent to the detection loss for the traditional method [see Eq. (8) in Sec. II C]. Therefore, it is crucial to ensure that the coupling efficiency between the entangled source and PSA is as high as possible.

\section{MEASUREMENT OF MULTIMODE ENTANGLED STATES}

In previous sections, each field of the entangled source is viewed as in single mode. In practice, we always have to deal with systems in multiple modes. Particularly, for the entangled source generated in pulse-excited system, each field of the source is composed of multiple-frequency components. In this section, we will extend the models of entangled sources and measurement schemes from single-mode case to multimode case. After briefly describing the process of measuring multimode entanglement by using the traditional method depicted in Fig. 1, we will analyze the performance of our method and show its advantages in measuring multitemporal mode entangled states.

\section{A. Entanglement generated from pulse pumped parametric amplifier}

If the entangled source in Fig. 1(a) is realized by pumping the PA with a train of short laser pulses, the two output fields will be of multimode nature [21]. However, no matter how complicated the system is, a parametric amplifier, when treated as a linear device, can always be viewed as superposition of its eigenmodes, which do not change after the amplifier. Assuming the nonlinear medium of parametric amplifier is in waveguide structure and only supports a single spatial mode, there exists an independent set of pairwise temporal modes $\left\{\hat{A}_{j}, \hat{B}_{j}\right\}(j=1,2, \ldots)$ for the entangled fields, and the input-output relation can be expressed as $[31,37]$

$$
\begin{aligned}
& \hat{A}_{j}=\mu_{j} \hat{A}_{j}^{\mathrm{in}}+v_{j} \hat{B}_{j}^{\mathrm{in} \dagger}, \\
& \hat{B}_{j}=\mu_{j} \hat{B}_{j}^{\mathrm{in}}+v_{j} \hat{A}_{j}^{\mathrm{in} \dagger},
\end{aligned}
$$

where

$$
\begin{aligned}
\hat{A}_{j}^{\dagger} & \equiv \int d \omega_{1} \phi_{j}\left(\omega_{1}\right) \hat{a}_{1}^{\dagger}\left(\omega_{1}\right), \\
\hat{B}_{j}^{\dagger} & \equiv \int d \omega_{2} \psi_{j}\left(\omega_{2}\right) \hat{a}_{2}^{\dagger}\left(\omega_{2}\right)
\end{aligned}
$$

define the creation operators of two entangled fields $\hat{a}_{1}$ and $\hat{a}_{2}$, and $\mu_{j}, v_{j}$ with $\mu_{j}^{2}-v_{j}^{2}=1\left(\mu_{1} \geqslant \mu_{2} \geqslant \mu_{3} \ldots\right)$ are the gain coefficient for $j$ th mode. The complex functions $\phi_{j}\left(\omega_{1}\right)$ and $\psi_{j}\left(\omega_{2}\right)$ satisfying the orthonormal relations, $\int d \omega_{1} \phi_{i}^{*}\left(\omega_{1}\right) \phi_{j}\left(\omega_{1}\right)=\delta_{i j}=\int d \omega_{2} \psi_{i}^{*}\left(\omega_{2}\right) \psi_{j}\left(\omega_{2}\right)$, represent the spectrum of two entangled fields in the $j$ th-order 
temporal modes $f_{j}(\tau)=\int d \omega_{1} \phi_{j}\left(\omega_{1}\right) e^{-i \omega_{1} \tau}$ and $h_{j}(\tau)=$ $\int d \omega_{2} \psi_{j}\left(\omega_{2}\right) e^{-i \omega_{2} \tau}$.

The noise variances for the difference and sum of quadrature amplitude of $j$ th mode are

$$
\begin{aligned}
\left\langle\Delta^{2} \hat{X}_{-}\right\rangle_{j} & =\left\langle\Delta^{2}\left(\hat{X}_{1 j}-\hat{X}_{2 j}\right)\right\rangle=2\left(\mu_{j}-v_{j}\right)^{2}, \\
\left\langle\Delta^{2} \hat{Y}_{+}\right\rangle_{j} & =\left\langle\Delta^{2}\left(\hat{Y}_{1 j}+\hat{Y}_{2 j}\right)\right\rangle=2\left(\mu_{j}-v_{j}\right)^{2},
\end{aligned}
$$

where $\hat{X}_{j}=\hat{A}_{j}+\hat{A}_{j}^{\dagger}, \hat{Y}_{j}=-i\left(\hat{A}_{j}-\hat{A}_{j}^{\dagger}\right)$ are the quadraturephase amplitudes for the $j$ th modes. The corresponding SNL for each mode

$$
\left\langle\Delta^{2} \hat{X}_{-}\right\rangle_{j-\mathrm{SNL}}=\left\langle\Delta^{2} \hat{Y}_{+}\right\rangle_{j-\mathrm{SNL}}=2
$$

can be obtained by placing the output fields $\hat{a}_{1}, \hat{a}_{2}$ in vacuum.

For the two entangled fields in $j$ th mode pair, $\phi_{j}\left(\omega_{1}\right)$ and $\psi_{j}\left(\omega_{2}\right)$, the normalized noise variances and inseparability of the source are written as

$$
\begin{gathered}
\left\langle\Delta^{2} \hat{X}_{-}\right\rangle_{j-s}=\frac{\left\langle\Delta^{2} \hat{X}_{-}\right\rangle_{j}}{\left\langle\Delta^{2} \hat{X}_{-}\right\rangle_{j-\mathrm{SNL}}}=\left(\mu_{j}-v_{j}\right)^{2}, \\
\left\langle\Delta^{2} \hat{Y}_{+}\right\rangle_{j-s}=\frac{\left\langle\Delta^{2} \hat{Y}_{+}\right\rangle_{j}}{\left\langle\Delta^{2} \hat{Y}_{+}\right\rangle_{j-\mathrm{SNL}}}=\left(\mu_{j}-v_{j}\right)^{2}, \\
\text { and } I_{j-s}=\left\langle\Delta^{2} \hat{X}_{-}\right\rangle_{j-s}+\left\langle\Delta^{2} \hat{Y}_{+}\right\rangle_{j-s}=2\left(\mu_{j}-v_{j}\right)^{2},
\end{gathered}
$$

where subscript $j-s$ indicates the contribution from the $j$ th mode of the EPR source.

\section{B. Measuring pulsed entanglement with the traditional method}

When the correlation between two pulsed fields is measured by the traditional method in Fig. 1, the fields of LOs for two BHDs, $A_{\mathrm{LO}}(t), B_{\mathrm{LO}}(t)$, should be in pulsed form as well. If the two LOs are transform-limited pulses, we have

$$
\begin{aligned}
& A_{\mathrm{LO}}(t)=\mathcal{E} e^{i \phi_{0}} \frac{1}{\sqrt{2 \pi}} \int d \omega_{1} A_{\mathrm{LO}}\left(\omega_{1}\right) e^{-i \omega_{1} t}, \\
& B_{\mathrm{LO}}(t)=\mathcal{E} e^{i \psi_{0}} \frac{1}{\sqrt{2 \pi}} \int d \omega_{2} B_{\mathrm{LO}}\left(\omega_{2}\right) e^{-i \omega_{2} t},
\end{aligned}
$$

where the frequency components $A_{\mathrm{LO}}\left(\omega_{1}\right), B_{\mathrm{LO}}\left(\omega_{2}\right)$ satisfy the normalization condition $\int d \omega_{1}\left|A_{\mathrm{LO}}\left(\omega_{1}\right)\right|^{2}=1$ and $\int d \omega_{2}\left|B_{\mathrm{LO}}\left(\omega_{2}\right)\right|^{2}=1, \phi_{0}, \psi_{0}$ are the global phases of the two LOs, and $\mathcal{E}$ denotes amplitude of each LO. When the amplitude of each LO is much stronger than the detected fields, i.e., $\mathcal{E} \gg 1$, the output currents of two BHDs are given by

$$
\begin{aligned}
& \hat{i}_{1}=\int d t\left[A_{\mathrm{LO}}^{*}(t) \hat{E}_{1}(t)+\text { H.c. }\right], \\
& \hat{i}_{2}=\int d t\left[B_{\mathrm{LO}}^{*}(t) \hat{E}_{2}(t)+\text { H.c. }\right],
\end{aligned}
$$

where $\hat{E}_{1,2}(t)=\frac{1}{\sqrt{2 \pi}} \int d \omega \hat{a}_{1,2}(\omega) e^{-i \omega t}$ are the field operators for the detected fields.
With the orthogonal modes defined in Eq. (45), the LO fields can be decomposed as

$$
\begin{aligned}
& A_{\mathrm{LO}}(\omega)=\sum_{j} \xi_{j} \phi_{j}(\omega), \\
& B_{\mathrm{LO}}(\omega)=\sum_{j} \zeta_{j} \psi_{j}(\omega),
\end{aligned}
$$

where

$$
\begin{gathered}
\xi_{j} \equiv\left|\xi_{j}\right| e^{i \theta_{j}}=\int d \omega A_{\mathrm{LO}}(\omega) \phi_{j}^{*}(\omega), \\
\zeta_{j} \equiv\left|\zeta_{j}\right| e^{i \theta_{j}^{\prime}}=\int d \omega B_{\mathrm{LO}}(\omega) \psi_{j}^{*}(\omega)
\end{gathered}
$$

with $\sum_{j}\left|\xi_{j}\right|^{2}=1=\sum_{j}\left|\zeta_{j}\right|^{2}$ are the complex coefficients characterizing the mode matching. $\theta_{j}, \theta_{j}^{\prime}$ are the LO phases for measuring the two entangled fields in $j$ th mode. Accordingly, the output currents in Eq. (51) can be rewritten as

$$
\begin{aligned}
& \hat{i}_{1}=\mathcal{E} \sum_{j}\left|\xi_{j}\right| \hat{X}_{1 j}\left(\theta_{j}+\phi_{0}\right), \\
& \hat{i}_{2}=\mathcal{E} \sum_{j}\left|\zeta_{j}\right| \hat{X}_{2 j}\left(\theta_{j}^{\prime}+\psi_{0}\right),
\end{aligned}
$$

where $\hat{X}_{1 j}\left(\theta_{j}\right) \equiv \hat{A}_{j} e^{-i \theta_{j}}+\hat{A}_{j}^{\dagger} e^{i \theta_{j}}, \hat{X}_{2 j}\left(\theta_{j}^{\prime}\right) \equiv \hat{B}_{j} e^{-i \theta_{j}^{\prime}}+\hat{B}_{j}^{\dagger} e^{i \theta_{j}^{\prime}}$ are the quadrature-phase amplitudes for the $j$ th mode. To obtain the quantum correlation between the quadrature amplitudes of two fields, we measure variance in current difference and sum

$$
\begin{aligned}
\left\langle\Delta^{2}\left(\hat{i}_{1} \mp \hat{i}_{2}\right)\right\rangle= & \mathcal{E}^{2} \sum_{j}\left\langle\Delta ^ { 2 } \left[\left|\xi_{j}\right| \hat{X}_{1 j}\left(\theta_{j}+\phi_{0}\right)\right.\right. \\
& \left.\left.\mp\left|\zeta_{j}\right| \hat{X}_{2 j}\left(\theta_{j}^{\prime}+\psi_{0}\right)\right]\right\rangle .
\end{aligned}
$$

It is well known that the entanglement degree, reflected by the inseparability, is defined through a definite quadraturephase amplitude and its orthogonal component of two fields, and the measurement output depends on different quadraturephase angles. So we set the LO phases at two sets of orthogonal angles: $\phi_{0}, \psi_{0}$ and $\phi_{0}+\pi / 2, \psi_{0}-\pi / 2$. We then investigate how to minimize the calculated inseparability by changing $\phi_{0}, \psi_{0}$.

For the entangled fields described in Eq. (44), we calculate the inseparability from the traditional method through the quantity in Eq. (55) and the definition of inseparability in Eq. (3) leads to

$$
I^{\text {multi }}=\frac{1}{2} \sum_{j} I_{j}
$$

with $I_{j} \equiv\left\langle\Delta^{2}\left[\left|\xi_{j}\right| \hat{X}_{1 j}\left(\theta_{j}+\phi_{0}\right)-\left|\zeta_{j}\right| \hat{X}_{2 j}\left(\theta_{j}^{\prime}+\psi_{0}\right)\right]\right\rangle+\left\langle\Delta^{2}\left[\left|\xi_{j}\right|\right.\right.$ $\left.\left.\hat{X}_{1 j}\left(\theta_{j}+\phi_{0}+\pi / 2\right)-\left|\zeta_{j}\right| \hat{X}_{2 j}\left(\theta_{j}^{\prime}+\psi_{0}-\pi / 2\right)\right]\right\rangle$. Note that for the single-mode case, we have $\xi_{j}=\delta_{j, j_{0}}=\zeta_{j}$ and we can recover the expression for inseparability in Eq. (3). For the multimode entangled source described by Eq. (44), we find

$$
\begin{aligned}
I_{j} & =2\left|\mu_{j} \xi_{j}-v_{j} \zeta_{j}^{*} e^{-i\left(\phi_{0}+\psi_{0}\right)}\right|^{2}+2\left|v_{j} \xi_{j}-\mu_{j} \zeta_{j}^{*} e^{-i\left(\phi_{0}+\psi_{0}\right)}\right|^{2} \\
& =2\left(\mu_{j}^{2}+v_{j}^{2}\right)\left(\left|\xi_{j}\right|^{2}+\left|\zeta_{j}\right|^{2}\right)-8 \mu_{j} v_{j}\left|\xi_{j}\right|\left|\zeta_{j}\right| \cos \Delta \theta_{j}
\end{aligned}
$$


with $\Delta \theta_{j} \equiv \theta_{j}+\theta_{j}^{\prime}+\phi_{0}+\psi_{0}$. If the LOs match one specific pair of modes of the entangled fields, labeled as $j_{0}$ th mode, we have $\xi_{j}=\delta_{j j_{0}}=\zeta_{j}$ and $I^{\text {multi }}=2\left(\mu_{j_{0}}-v_{j_{0}}\right)^{2}$. This is the same as the single mode case discussed earlier. Otherwise, we need to sum up all terms given in Eq. (57). Although $\theta_{j}$ and $\theta_{j}^{\prime}$ vary with $j$, the sum $\theta_{j}+\theta_{j}^{\prime}$ usually inherits the phase of the pump and changes little with $j$. So we can always choose $\phi_{0}+\psi_{0}$ so that $\Delta \theta_{j}=0$ for all $j$. In this case, the phases of different modes are synchronized and the inseparability obtained from homodyne detection is

$$
I^{\text {multi }}=\sum_{j}\left[\left(\mu_{j}\left|\xi_{j}\right|-v_{j}\left|\zeta_{j}\right|\right)^{2}+\left(v_{j}\left|\xi_{j}\right|-\mu_{j}\left|\zeta_{j}\right|\right)^{2}\right],
$$

which can be viewed as an averaged value of inseparability of each mode pair.

However, due to dispersion and birefringence in the nonlinear medium for parametric amplifier, the sum $\theta_{j}+\theta_{j}^{\prime}$ may change with $j$ and it is thus impossible to have $\Delta \theta_{j}=0$ for all $j$, leading to asynchronized phases for different modes. This effect is detrimental for traditional method of inseparability measurement because $I_{j}$ cannot reach minimum value at the same $\phi_{0}+\psi_{0}$ : While $I_{j}$ is minimum for some $j$, it is higher for other $j$, leading to higher $I^{\text {multi }}$ than what is expected from the optimum value in Eq. (58).

In addition to the asynchronized phase problem, the mode shapes of the LOs are also critical in BHD for pulsed case. For the pulsed pump with Gaussian-shaped spectrum, the gain coefficient of the fundamental mode $v_{j}$ with $j=1$ is the highest [38]. If we can perfectly match the modes of LOs with the two entangled fields in fundamental modes, we will be able to obtain the lowest value of inseparability coefficient, $I_{1}$, i.e., $I_{1} \leqslant I^{\text {multi }}$. However, the method of precisely knowing the temporal-mode profile has not been available yet, so it is difficult to directly measure $I_{1}$ by properly shaping the spectrum of LOs. When the mode-matching coefficients for the two entangled fields $\xi_{j}$ and $\zeta_{j}$ are significantly different from each other, the measured value of $I^{\text {multi }}$ may become higher than 2 due to the thermal nature of individual fields. For example, assuming the entangled source is a superposition of two pairs of temporal modes, if the mode-matching coefficients between the detected fields and local oscillators are $\left|\xi_{1}\right|=0,\left|\xi_{2}\right|=1$ and $\left|\zeta_{1}\right|=1,\left|\zeta_{2}\right|=0$, the measured value of $I^{\text {multi }}=\mu_{1}^{2}+\mu_{2}^{2}+v_{1}^{2}+v_{2}^{2}$ is higher than 2 , and the inseparability criterion cannot be obtained. So the entanglement degree of pulsed entangled source measured by traditional method is always smaller than what is anticipated. Next, we will analyze the performance of our method with PSA in measuring pulsed entanglement.

\section{Measurement using the PSA-assisted balanced homodyne detections}

To clearly illustrate the advantage of our method in a multimode case, we first take the PSA-assisted scheme in Fig. 5 as an example. Assuming the PSA has the same spectrum property as the entangled source but has a different set of gains, $G_{j}, g_{j}$, that is, they have the same mode structure $\left\{\phi_{j}, \psi_{j}\right\}$. This is the case if the two parametric amplifiers are from the same nonlinear process. From mode decomposition of the pulse-pumped parametric proess [31,37], we know that
$G_{j}=\cosh \left(r_{j} G^{\prime}\right), g_{j}=\sinh \left(r_{j} G^{\prime}\right)$ with $r_{1}>r_{2}>\ldots$ as the mode number and $\sum r_{j}^{2}=1$, where $G^{\prime}$ is the gain parameter related to the pump power. The input-output relation for the PSA is similar to Eq. (44) but with $\mu_{j}, v_{j}$ replaced by the PSA's gain parameters $G_{j},-g_{j}$. Here, we choose $-g_{j}$ to describe the PSA in deamplification.

Assuming the PSA gain is large $\left(G_{j} \approx g_{j} \gg 1\right)$, we find the measured fluctuations of the quadrature-phase amplitudes of each mode at two outputs of the amplifier can be respectively written as

$$
\begin{aligned}
\left\langle\Delta^{2} \hat{X}_{1 j}^{\text {out }}\left(\theta_{j}+\phi_{0}\right)\right\rangle & =2\left|\xi_{j}\right|^{2} G_{j}^{2}\left(\mu_{j}-v_{j}\right)^{2}, \\
\left\langle\Delta^{2} \hat{X}_{2 j}^{\text {out }}\left(\theta_{j}^{\prime}+\psi_{0}\right)\right\rangle & =2\left|\zeta_{j}\right|^{2} G_{j}^{2}\left(\mu_{j}-v_{j}\right)^{2} .
\end{aligned}
$$

The SNL measured at $\hat{a}_{1}^{\text {out }}$ is $\left\langle\Delta^{2} \hat{X}_{1 j}^{\text {out }}\left(\theta_{j}+\phi_{0}\right)\right\rangle_{\mathrm{SNL}}=$ $2 \sum_{j}\left|\xi_{j}\right|^{2} G_{j}^{2}$, so the normalized values $\left\langle\Delta^{2} \hat{X}_{1 j}^{\text {out }}\right\rangle_{N o r}$ and $\left\langle\Delta^{2} \hat{Y}_{1 j}^{\text {out }}\right\rangle_{N o r}$ are

$$
\left\langle\Delta^{2} \hat{X}_{1 j}^{\text {out }}\right\rangle_{N o r}=\left\langle\Delta^{2} \hat{Y}_{1 j}^{\text {out }}\right\rangle_{N o r}=\frac{\sum_{j}\left|\xi_{j}\right|^{2} G_{j}^{2}\left(\mu_{j}-v_{j}\right)^{2}}{\sum_{j}\left|\xi_{j}\right|^{2} G_{j}^{2}} .
$$

As a result, the inseparability measured from output port $\hat{a}_{1}^{\text {out }}$ is

$$
I_{\mathrm{amp}}^{\text {multi }}=\frac{2 \sum_{j}\left|\xi_{j}\right|^{2} G_{j}^{2}\left(\mu_{j}-v_{j}\right)^{2}}{\sum_{j}\left|\xi_{j}\right|^{2} G_{j}^{2}} .
$$

Note that the expression above does not show the asynchronized phase problem: All modes reaches the minimum value together. For the output $\hat{a}_{2}^{\text {out }}$ of PSA, the measured inseparability is similar as Eq. (61) but replacing $\xi_{j}$ with $\zeta_{j}$. Since $r_{1}>$ $r_{2}>\ldots$, we have $G_{1}=\cosh \left(r_{1} G^{\prime}\right) \gg \cosh \left(r_{2} G^{\prime}\right)=G_{2} \gg$ $G_{3} \ldots$ For $G^{\prime} \rightarrow \infty$, Eq. (61) can be approximated as

$$
I_{\text {amp }}^{\text {multi }} \approx \frac{2\left|\xi_{1}\right|^{2} G_{1}^{2}\left(\mu_{1}-v_{1}\right)^{2}}{\left|\xi_{1}\right|^{2} G_{1}^{2}}=2\left(\mu_{1}-v_{1}\right)^{2} .
$$

Similarly, for the PSA-assisted joint measurement in Fig. 4, the inseparability is

$$
I_{\text {amp }-J M}^{\text {multi }}=\frac{2 \sum_{j}\left(\left|\xi_{j}\right|+\left|\zeta_{j}\right|\right)^{2} G_{j}^{2}\left(\mu_{j}-v_{j}\right)^{2}}{\sum_{j}\left(\left|\xi_{j}\right|+\left|\zeta_{j}\right|\right)^{2} G_{j}^{2}}
$$

for PSA with large gain. Since $r_{1}>r_{2}>\ldots$, Eq. (63) can be approximated as

$$
I_{\text {multi }}^{\text {amp- } J M} \approx \frac{2\left(\left|\xi_{1}\right|+\left|\zeta_{1}\right|\right)^{2} G_{1}^{2}\left(\mu_{1}-v_{1}\right)^{2}}{\mid\left(\left|\xi_{1}\right|+\left|\zeta_{1}\right|\right)^{2} G_{1}^{2}}=2\left(\mu_{1}-v_{1}\right)^{2}
$$

for $G^{\prime} \rightarrow \infty$. Comparing Eqs. (62) and (64) with Eqs. (61), (63), and (58), one sees that the high-gain PSA can select out the mode pair with $j=1$ from the entangled fields. Only $j=1$ modes contribute to the measurement results as if it were the single temporal mode case. By properly setting the LO phases of BHDs, the variances $\left\langle\Delta^{2} \hat{X}_{-}\right\rangle_{1-s}$, $\left\langle\Delta^{2} \hat{Y}_{+}\right\rangle_{1-s}$ and inseparability $I_{1-s}$ can be measured, and the results are immune to detection loss [39]. Therefore, the high-gain PSA-assisted BHD is better than the traditional BHD method [39]. So, this measurement method is more advantageous than the traditional method in the multimode case. If there is mode mismatching between PSA and 
entangled source PA, then the degree of mode mismatching can be viewed as the coupling loss between them, whose effect on the measurement is similar to that discussed in the end of Sec. IV D.

\section{SUMMARY AND DISCUSSION}

In summary, we have investigated a method of detecting continuous variable entanglement by using phasesensitive amplifier-assisted balanced homodyne detections. This method is robust in a number of unflavored situations such as the imperfect detection process of quantum states, namely nonunit quantum efficiency detectors and mode mismatch between the detected multimode fields and the local oscillators. Additionally, this method allows us to measure quantum entanglement even when one of the fields is not experimentally accessible, which effectively simplifies the experimental resources required by the traditional balanced homodyne measurement. A slight variation of the method also can measure $\hat{X}_{-}$and $\hat{Y}_{+}$at the same time, making it possible to measure phase and amplitude simultaneously. Moreover, it has the potential to tremendously increase the bandwidth in measuring the quantum correlation. This method is applicable to many relevant problems in quantum optics.

This method for measuring quantum entanglement should also be beneficial in areas such as quantum information and quantum metrology, where entangled quantum states are used for performance enhancement. Although we have only discussed the noise measurement, it works equally well when the input fields are encoded with modulation signals. Indeed, the underlying physics in the experiments of using $\mathrm{SU}(1,1)$ nonlinear interferometer for quantum metrology is that the advantage of entangled sources is maintained for the enhancement of the signal-to-noise ratio even in the presence of losses at detection $[31,37,40]$. This is the same as our method.
In this paper, we have studied this measurement method by taking two entangled fields having positive correlation between $\hat{X}_{1}$ and $\hat{X}_{2}$ and negative correlation between $\hat{Y}_{1}$ and $\hat{Y}_{2}$ as an example. In addition, we assume the correlation between two entangled fields is symmetric. We believe this method is suitable for measuring various kind of entanglement. For example, another type of entanglement with negative correlation between $\hat{X}_{1}$ and $\hat{X}_{2}$ and positive correlation between $\hat{Y}_{1}$ and $\hat{Y}_{2}$ [3] can also be measured by our method, but the PSA needs to be operated at the amplification condition, at which the noise variance at each output of PSA is proportional to product between the PSA gain $G^{2}$ and the variances of operators $\hat{X}_{1}+\hat{X}_{2}$ (or $\hat{Y}_{1}-\hat{Y}_{2}$ ). Moreover, for the entanglement with asymmetric correlation between two fields, $\hat{X}_{1} \mp k \hat{X}_{2}$ and $\hat{Y}_{1} \pm k \hat{Y}_{2}(k \neq 1)$, which can be generated from the entangled source with unbalanced internal losses in two fields, we can optimize the joint measurement by properly changing the electronic gain to adjust the photocurrent level out of one BHD. If only one BHD is placed at one output of PSA, the optimized measurement can be obtained by properly changing the gain of PSA.

Finally, it is worth pointing out that although this method of assisting BHD with high-gain PSA is useful in measuring the noise reduction and inseparability of entanglement, the PSA, which functions as a homodyne detection for realizing the measurement, it cannot be viewed as a tool for quantum state transformation. This is because the noise variances for the difference and sum of the quadrature amplitudes of the PSA output fields are not lower than vacuum noise, especially for the PSA with high gain, as illustrated by Eq. (22).

\section{ACKNOWLEDGMENT}

The work is supported in part by the National Natural Science Foundation of China (Grants No. 91836302, No. 91736105, and No. 11527808).
[1] S. L. Braunstein and H. J. Kimble, Phys. Rev. A 61, 042302 (2000).

[2] J. Zhang and K. Peng, Phys. Rev. A 62, 064302 (2000).

[3] X. Li, Q. Pan, J. Jing, J. Zhang, C. Xie, and K. Peng, Phys. Rev. Lett. 88, 047904 (2002).

[4] S. Steinlechner, J. Bauchrowitz, M. Meinders, H. MullerEbhardt, K. Danzmann, and R. Schnabel, Nat. Photon. 7, 626 (2013).

[5] C. H. Bennett, G. Brassard, C. Crépeau, R. Jozsa, A. Peres, and W. K. Wootters, Phys. Rev. Lett. 70, 1895 (1993).

[6] D. Bouwmeester, J.-W. Pan, K. Mattle, M. Eibl, H. Weinfurter, and A. Zeilinger, Nature (London) 390, 575 (1997).

[7] L. Vaidman, Phys. Rev. A 49, 1473 (1994).

[8] S. L. Braunstein and H. J. Kimble, Phys. Rev. Lett. 80, 869 (1998).

[9] A. Furusawa, J. L. Srensen, S. L. Braunstein, C. A. Fuchs, H. J. Kimble, and E. S. Polzik, Science 282, 706 (1998).

[10] A. N. Boto, P. Kok, D. S. Abrams, S. L. Braunstein, C. P. Williams, and J. P. Dowling, Phys. Rev. Lett. 85, 2733 (2000).
[11] V. Giovannetti, S. Lloyd, and L. Maccone, Phys. Rev. Lett. 96, 010401 (2006).

[12] Y. Ma, H. Miao, B. H. Pang, M. Evans, C. Zhao, J. Harms, R. Schnabel, and Y. Chen, Nat. Phys. 13, 776 (2017).

[13] W. Vogel, Phys. Rev. A 51, 4160 (1995).

[14] E. Shchukin and W. Vogel, Phys. Rev. Lett. 96, 200403 (2006).

[15] B. Kuhn, W. Vogel, M. Mraz, S. Kohnke, and B. Hage, Phys. Rev. Lett. 118, 153601 (2017).

[16] M. G. Raymer, J. Mod. Opt. 51, 1739 (2004).

[17] C.-L. Bian, L.-Q. Chen, G.-W. Zhang, Z. Y. Ou, and W. Zhang, Europhys. Lett. 97, 34005 (2012).

[18] B. Chen, C. Qiu, S. Chen, J. Guo, L. Q. Chen, Z. Y. Ou, and W. Zhang, Phys. Rev. Lett. 115, 043602 (2015).

[19] Y. Shaked, Y. Michael, R. Z. Vered, L. Bello, M. Rosenbluh, and A. Pe'er, Nat. Commun. 9, 609 (2018).

[20] E. Flurin, N. Roch, F. Mallet, M. H. Devoret, and B. Huard, Phys. Rev. Lett. 109, 183901 (2012). 
[21] W. Wasilewski, A. I. Lvovsky, K. Banaszek, and C. Radzewicz, Phys. Rev. A 73, 063819 (2006).

[22] J. Wenger, R. Tualle-Brouri, and P. Grangier, Opt. Lett. 29, 1267 (2004).

[23] Y. Eto, T. Tajima, Y. Zhang, and T. Hirano, Opt. Express 16, 10650 (2008).

[24] X. Guo, N. Liu, Y. Liu, X. Li, and Z. Y. Ou, Opt. Lett. 41, 653 (2016).

[25] A. Einstein, B. Podolsky, and N. Rosen, Phys. Rev. 47, 777 (1935).

[26] M. D. Reid, Phys. Rev. A 40, 913 (1989).

[27] Z. Y. Ou, S. F. Pereira, H. J. Kimble, and K. C. Peng, Phys. Rev. Lett. 68, 3663 (1992).

[28] L.-M. Duan, G. Giedke, J. I. Cirac, and P. Zoller, Phys. Rev. Lett. 84, 2722 (2000).

[29] R. E. Slusher, P. Grangier, A. LaPorta, B. Yurke, and M. J. Potasek, Phys. Rev. Lett. 59, 2566 (1987).

[30] O. Aytür and P. Kumar, Phys. Rev. Lett. 65, 1551 (1990).
[31] X. Guo, N. Liu, X. Li, and Z. Y. Ou, Opt. Express 23, 29369 (2015).

[32] C. M. Caves, J. Combes, Z. Jiang, and S. Pandey, Phys. Rev. A 86, 063802 (2012).

[33] J. Xin, J. Qi, and J. Jing, Opt. Lett. 42, 366 (2017).

[34] J. Li, Y. Liu, L. Cui, N. Huo, S. M. Assad, X. Li, and Z. Y. Ou, Phys. Rev. A 97, 052127 (2018).

[35] Z. Y. Ou, Phys. Rev. A 85, 023815 (2012).

[36] Y. Liu, J. Li, L. Cui, N. Huo, S. M. Assad, X. Li, and Z. Y. Ou, Opt. Express 26, 27705 (2018).

[37] A. Christ, K. Laiho, A. Eckstein, K. N. Cassemiro, and C. Silberhorn, New J. Phys. 13, 033027 (2011).

[38] N. Liu, Y. Liu, X. Guo, L. Yang, X. Li, and Z. Y. Ou, Opt. Express 24, 1096 (2016).

[39] J. Li, Y. Liu, N. Huo, L. Cui, C. Feng, Z. Y. Ou, and X. Li, Opt. Express 27, 30552 (2019).

[40] Y. Liu, N. Huo, J. Li, L. Cui, X. Li, and Z. J. Ou, Opt. Express 27, 11292 (2019). 